\title{
Biaryl Synthesis via Direct Arylation: Establishment of an Efficient Catalyst for Intramolecular Processes
}

\author{
Louis-Charles Campeau, Mathieu Parisien, Melissa Leblanc \& Keith Fagnou* \\ Center for Catalysis Research and Innovation, University of Ottawa, Department \\ of Chemistry, 10 Marie Curie, Ottawa, Ontario, Canada K1N 6N5 \\ Keith.Fagnou@science.uottawa.ca
}

\section{Supporting Information}

General Methods. All experiments were carried out under an atmosphere of nitrogen. ${ }^{1} \mathrm{H}$ and ${ }^{13} \mathrm{C}$ NMR were recorded in $\mathrm{CDCl}_{3}$ solutions using a Bruker AVANCE 300 spectrometer with $\mathrm{Me}_{4} \mathrm{Si}$ as an internal standard. High-resolution mass spectra were obtained on a Kratos Concept IIH. Infra-Red analysis was performed with a Bruker EQUINOX 55. Unless otherwise specified, all reagents and solvents were used as is from commercial sources.

Bromo ethers 7 and $15^{1}, 11^{2}, 3^{3}$ were prepared according to the general procedure and exhibited spectral data identical to literature values ${ }^{1-3}$ Cyclization precursors $21^{4}$ and $19^{5}$ were prepared according, and exhibited spectral data identical, to literature. ${ }^{4-5}$

General procedure for the synthesis of bromo ethers $3,7,9,11,13,15,17$ :

To a mixture of $\mathrm{K}_{2} \mathrm{CO}_{3}(4.4 \mathrm{~g}, 32 \mathrm{mmol}$, 2eq.) and 4-chloro phenol $(6.2 \mathrm{~g}$, $48 \mathrm{mmol}$, 3eq.) was added $30 \mathrm{~mL}$ of acetone in a $100 \mathrm{~mL}$ round bottom flask equipped with a mechanical stir bar. To the stirring mixture was added 2bromobenzyl bromide $\left(4 \mathrm{~g}, 16 \mathrm{mmol}\right.$, 1eq.) followed by heating to $50^{\circ} \mathrm{C}$ overnight. The reaction mixture was then poured into a $\mathrm{NaOH}(2 \mathrm{~N})$ solution and extracted with ethyl acetate. The organic extracts were dried over $\mathrm{MgSO}_{4}$, and concentrated under reduced pressure. Purification was done by flash chromatography using $15 \%$ ethyl acetate in hexanes to afford (2-Bromo phenyl)methyl 4-chlorophenyl ether $\mathbf{1 5}^{\mathbf{1}}$, as a clear oil in $90 \%$ yield $(4.27 \mathrm{~g})$.

1-(2-Bromo-benzyloxy)-p-toluene (9)

$R_{\mathrm{f}}=0.64$ on silica gel (5\% EtOAc:Hexanes); IR $\left(v_{\max } / \mathrm{cm}^{-1}\right) 3029$ (weak), 1510, 1237, 1024, 816, 745 ; ${ }^{1} \mathrm{H}$ NMR $\left(300 \mathrm{MHz}, \mathrm{CDCl}_{3}, 293 \mathrm{~K}, \mathrm{TMS}\right): 2.26(3 \mathrm{H}, \mathrm{s}), 5.06$ $(2 \mathrm{H}, \mathrm{s}), 6.85(2 \mathrm{H}, \mathrm{m}), 7.10(3 \mathrm{H}, \mathrm{m}), 7.27(1 \mathrm{H}, \mathrm{td}, \mathrm{J}=8 \mathrm{~Hz} \& \mathrm{~J}=1 \mathrm{~Hz}), 7.52(2 \mathrm{H}, \mathrm{m})$; ${ }^{13} \mathrm{C}$ NMR $\left(75 \mathrm{MHz}, \mathrm{CDCl}_{3}, 293 \mathrm{~K}, \mathrm{TMS}\right): 20.47,69.37,114.65,122.17,127.46$, 128.78, 129.04, 129.93, 130.32, 132.49, 136.49, 156.29; HRMS calculated for $\mathrm{C}_{14} \mathrm{H}_{13} \mathrm{BrO}(\mathrm{M}+)$ 276.0163; Found : 276.0150

1-(2-Bromo-benzyloxy)-a,a, $\alpha$-trifluorotoluene (13)

$\mathrm{R}_{\mathrm{f}}=0.62$ on silica gel (5\% EtOAc:Hexanes); IR $\left(v_{\max } / \mathrm{cm}^{-1}\right) 2925$ (weak), 1320, 1248, 1108, 841, $750 ;{ }^{1} \mathrm{H}$ NMR $\left(300 \mathrm{MHz}, \mathrm{CDCl}_{3}, 293 \mathrm{~K}, \mathrm{TMS}\right): 5.14(2 \mathrm{H}, \mathrm{s})$, $7.02(2 \mathrm{H}, \mathrm{d}, \mathrm{J}=9 \mathrm{~Hz}), 7.17(1 \mathrm{H}, \mathrm{t}, \mathrm{J}=8 \mathrm{~Hz}), 7.31(1 \mathrm{H}, \mathrm{d}, \mathrm{J}=7 \mathrm{~Hz}), 7.52(4 \mathrm{H}, \mathrm{m})$; 
${ }^{13} \mathrm{C}$ NMR (75MHz, $\left.\mathrm{CDCl}_{3}, 293 \mathrm{~K}, \mathrm{TMS}\right): 69.51,114.85,122.39,123.34$ (q, $J=33 \mathrm{~Hz}$ ), 124.28 (q, J=271Hz), 126.98 (q, $J=3 \mathrm{~Hz}), 127.68,128.87,129.56$, 132.77, 135.46. 160.82; HRMS calculated for $\mathrm{C}_{14} \mathrm{H}_{11} \mathrm{BrF}_{3} \mathrm{O}(\mathrm{M}+)$ 329.9853; Found: 329.9867

1-(2-Bromo-benzyloxy)-naphthalene (17)

$\mathrm{R}_{\mathrm{f}}=0.55$ on silica gel (5\% EtOAc:Hexanes); IR $\left(v_{\max } / \mathrm{cm}^{-1}\right) 3053$ (weak), 1364, 1246, 1096, $758 ;{ }^{1} \mathrm{H}$ NMR $\left(300 \mathrm{MHz}, \mathrm{CDCl}_{3}, 293 \mathrm{~K}, \mathrm{TMS}\right): 5.33(2 \mathrm{H}, \mathrm{s}), 6.89(1 \mathrm{H}$, $\mathrm{d}, J=8 \mathrm{~Hz}), 7.24(1 \mathrm{H}, \mathrm{d}, J=3 \mathrm{~Hz}), 7.38(2 \mathrm{H}, \mathrm{m}), 7.49(3 \mathrm{H}, \mathrm{m}), 7.62(1 \mathrm{H}, \mathrm{d}, J=8 \mathrm{~Hz})$, $7.69(1 \mathrm{H}, \mathrm{d}, \mathrm{J}=8 \mathrm{~Hz}), 7.82(1 \mathrm{H}, \mathrm{m}), 8.38(1 \mathrm{H}, \mathrm{m}) ;{ }^{13} \mathrm{C} \mathrm{NMR}\left(75 \mathrm{MHz}, \mathrm{CDCl}_{3}, 293 \mathrm{~K}\right.$, TMS) : $69.46,105.35,120.75,122.25,125.34,125.66,125.83,126.49,127.53$, 127.60, 128.72, 129.21, 132.64, 134.56, 136.38, 154.03; HRMS calculated for $\mathrm{C}_{17} \mathrm{H}_{13} \mathrm{BrO}(\mathrm{M}+)$ 312.0046; Found: 312.0150

\section{1-Bromo-2-(2-phenoxy-ethyl)-benzene (23)}

$\mathrm{R}_{\mathrm{f}}=0.50$ on silica gel (5\% EtOAc:Hexanes); IR $\left(V_{\max } / \mathrm{cm}^{-1}\right) 2938$ (weak), 1240, 1036, 747 ; ${ }^{1} \mathrm{H}$ NMR $\left(300 \mathrm{MHz}, \mathrm{CDCl}_{3}, 293 \mathrm{~K}, \mathrm{TMS}\right): 3.22(2 \mathrm{H}, \mathrm{t}, \mathrm{J}=7 \mathrm{~Hz}), 4.17$ $(2 \mathrm{H}, \mathrm{t}, \mathrm{J}=7 \mathrm{~Hz}), 6.91(3 \mathrm{H}, \mathrm{m}), 7.08(1 \mathrm{H}, \mathrm{m}), 7.26(4 \mathrm{H}, \mathrm{m}), 7.53(1 \mathrm{H}, \mathrm{dd}, \mathrm{J}=9 \mathrm{~Hz}$ \& $1 \mathrm{~Hz}) ;{ }^{13} \mathrm{C}$ NMR $\left(75 \mathrm{MHz}, \mathrm{CDCl}_{3}, 293 \mathrm{~K}, \mathrm{TMS}\right): 35.99,66.59,114.47,120.72$, $124.63,127.45,128.26,129.42,131.29,123.80,137.49,158.60$; HRMS calculated for $\mathrm{C}_{14} \mathrm{H}_{13} \mathrm{BrO}(\mathrm{M}+)$ 276.0135; Found: 276.0150

$6 H$-benzo[c]chromenes $8^{1}$ and $16^{1}, 10^{1}, 4^{3}$ were prepared according to the general procedure and exhibited spectral data identical to literature values. ${ }^{1,3}$ Cyclization product $22^{6}$ and $20^{5}$ as well as by product $5^{3}$ exhibited spectral data identical to literature values. ${ }^{3,4,6}$

\section{General procedure for cyclization:}

To a mixture of crushed $\mathrm{K}_{2} \mathrm{CO}_{3}(169 \mathrm{mg}, 1.22 \mathrm{mmol})$ and bromo ether 15 $(169 \mathrm{mg}, \quad 0.61 \mathrm{mmol})$ under nitrogen atmosphere was added $3 \mathrm{~mL}$ of $\mathrm{N}, \mathrm{N}$ Dimethylacetamide (DMA) in a $10 \mathrm{~mL}$ round bottom flask equipped with a mechanical stir bar. To the stirring reaction mixture was added $50 \mu \mathrm{L}$ of a $\mathrm{Pd}(\mathrm{OAc})_{2}$ and Ligand ${ }^{7}$ stock solution containing $7 \mathrm{mg} / \mathrm{mL} \mathrm{Pd}(\mathrm{OAc})_{2}$ and $36 \mathrm{mg} / \mathrm{mL}$ Ligand $^{7}$. (Similar results are obtained if the palladium and ligand are weighed out exactly and placed in the reaction mixture prior to addition the solvent). The reaction mixture is then heated overnight at $125^{\circ} \mathrm{C}$. After the reaction was judged complete by TLC or GC/MS analysis, the heat source was removed and the reaction mixture was allowed to cool. The crude mixture was then loaded onto a silica gel flash chromatography column $10 \%$ ethyl acetate in hexanes as the eluent to afford 2-Chloro- $6 \mathrm{H}$-benzo[c]chromene $16^{1}$ in $92 \%$ yield $(110 \mathrm{mg})$.

\section{4-Methyl-6H-benzo[c]chromene (12)}

$\mathrm{R}_{\mathrm{f}}=0.43$ on silica gel (5\% EtOAc:Hexanes); IR $\left(v_{\max } / \mathrm{cm}^{-1}\right) 2921$ (weak), 1421, 1249, 1195, 1020, 752; ${ }^{1} \mathrm{NMR}\left(300 \mathrm{MHz}, \mathrm{CDCl}_{3}, 293 \mathrm{~K}, \mathrm{TMS}\right): 2.26(3 \mathrm{H}, \mathrm{s}), 5.08$ $(2 \mathrm{H}, \mathrm{s}), 6.93(1 \mathrm{H}, \mathrm{t}, J=7 \mathrm{~Hz}), 7.09(2 \mathrm{H}, \mathrm{t}, J=7 \mathrm{~Hz}), 7.23(1 \mathrm{H}, \mathrm{t}, J=7 \mathrm{~Hz}), 7.32(1 \mathrm{H}, \mathrm{t}$, $J=3 \mathrm{~Hz}), 7.55(1 \mathrm{H}, \mathrm{d}, J=8 \mathrm{~Hz}), 7.64(1 \mathrm{H}, \mathrm{d}, J=8 \mathrm{~Hz}) ;{ }^{13} \mathrm{C} \mathrm{NMR}\left(75 \mathrm{MHz}, \mathrm{CDCl}_{3}\right.$, 
293K, TMS): 15.93, 68.33, 120.93, 121.41, 122.18, 122.41, 124.48, 126.64, $127.40,128.32,130.46,130.77,131.37,152.91$; HRMS calculated for $\mathrm{C}_{14} \mathrm{H}_{12} \mathrm{O}$ $(\mathrm{M}+)$ 196.0869; Found: 196.0888

\section{2-Trifluoromethyl-6H-benzo[c]chromene (14)}

$\mathrm{R}_{\mathrm{f}}=0.43$ on silica gel (5\% EtOAc:Hexanes); IR $\left(v_{\max } / \mathrm{cm}^{-1}\right) 2850$ (weak), 1315, 1250, 1112, $752{ }^{1} \mathrm{NMR}\left(300 \mathrm{MHz}, \mathrm{CDCl}_{3}, 293 \mathrm{~K}, \mathrm{TMS}\right): 5.12(2 \mathrm{H}, \mathrm{s}), 7.01(1 \mathrm{H}, \mathrm{d}$, $J=8 \mathrm{~Hz}), 7.11(1 \mathrm{H}, \mathrm{m}), 7.33(2 \mathrm{H}, \mathrm{m}), 7.44(1 \mathrm{H}, \mathrm{m}), 7.66(1 \mathrm{H}, \mathrm{d}, \mathrm{J}=7 \mathrm{~Hz}), 7.93(1 \mathrm{H}$, s); ${ }^{13} \mathrm{C}$ NMR (75MHz, $\left.\mathrm{CDCl}_{3}, 293 \mathrm{~K}, \mathrm{TMS}\right): 68.50,117.84,120.61$ (q, J=4Hz), 122.17, 123.07, 124.32 (q, J=33Hz), 124.46 (q, J=272Hz), 124.77, 126.30 (q, $\mathrm{J}=4 \mathrm{~Hz}), 128.56,128.73,130.98,157.22$; HRMS calculated for $\mathrm{C}_{14} \mathrm{H}_{9} \mathrm{~F}_{3} \mathrm{O}(\mathrm{M}+)$ 250.0601; Found: 250.0605

\section{H-Dibenzo[c, $h]$ chromene (18)}

$\mathrm{R}_{\mathrm{f}}=0.47$ on silica gel (5\% EtOAc:Hexanes); IR $\left(v_{\max } / \mathrm{cm}^{-1}\right) 2870$ (weak), 1395, 1351, 1096, 758; ${ }^{1} \mathrm{NMR}\left(300 \mathrm{MHz}, \mathrm{CDCl}_{3}, 293 \mathrm{~K}, \mathrm{TMS}\right): 5.23(2 \mathrm{H}, \mathrm{s}), 7.11(1 \mathrm{H}, \mathrm{d}$, $J=7 \mathrm{~Hz}), 7.22(1 \mathrm{H}, \mathrm{t}, \mathrm{J}=2 \mathrm{~Hz}), 7.33(1 \mathrm{H}, \mathrm{t}, \mathrm{J}=3 \mathrm{~Hz}), 7.45(3 \mathrm{H}, \mathrm{m}), 7.65(1 \mathrm{H}, \mathrm{d}$, $\mathrm{J}=3 \mathrm{~Hz}), 7.75(2 \mathrm{H}, \mathrm{m}), 8.25(1 \mathrm{H}, \mathrm{m}) ;{ }^{13} \mathrm{C} \mathrm{NMR}\left(75 \mathrm{MHz}, \mathrm{CDCl}_{3}, 293 \mathrm{~K}, \mathrm{TMS}\right)$ : $68.73,117.07,120.90,121.48,121.85,122.19,124.52,125.28,125.71,126.55$, 127.29, 127.57, 128.45, 130.56, 130.62, 134.29, 150.23; HRMS calculated for $\mathrm{C}_{17} \mathrm{H}_{12} \mathrm{O}(\mathrm{M}+)$ 232.0871; Found: 232.0888

\section{Dibenzoxapine (24)}

$\mathrm{R}_{\mathrm{f}}=0.36$ on silica gel (5\% EtOAc:Hexanes); IR $\left(v_{\max } / \mathrm{cm}^{-1}\right) 2870$ (weak), 1309, 1252, 1030, 758; ; ${ }^{1} \mathrm{NMR}\left(300 \mathrm{MHz}, \mathrm{CDCl}_{3}, 293 \mathrm{~K}, \mathrm{TMS}\right): 2.82(2 \mathrm{H}, \mathrm{t}, \mathrm{J}=6 \mathrm{~Hz})$, $4.57(2 \mathrm{H}, \mathrm{t}, \mathrm{J}=6 \mathrm{~Hz}), 7.14(1 \mathrm{H}, \mathrm{d}, \mathrm{J}=8 \mathrm{~Hz}), 7.29(4 \mathrm{H}, \mathrm{m}), 7.41(3 \mathrm{H}, \mathrm{m}) ;{ }^{13} \mathrm{C} \mathrm{NMR}$ $\left(75 \mathrm{MHz}, \mathrm{CDCl}_{3}, 293 \mathrm{~K}, \mathrm{TMS}\right): 33.39,78.42,122.31,124.55,127.35,127.71$, $128.03,128.13,129.02,129.21,135.24,137.37,138.86,154.33$; HRMS calculated for $\mathrm{C}_{14} \mathrm{H}_{12} \mathrm{O}(\mathrm{M}+)$ 196.0893; Found: 196.0888

\section{(1,1'-Biphenyl-2-yl)di(4-trifluoromethylphenyl)phosphine (27)}

$\mathrm{R}_{\mathrm{f}}=0.52$ on silica gel (5\% EtOAc:Hexanes); IR $\left(v_{\max } / \mathrm{cm}^{-1}\right) 2922$ (medium), $2860,1610,1318,1107,1064,1011,825,748,700 ;{ }^{1} \mathrm{H}$ NMR $\left(300 \mathrm{MHz}, \mathrm{CDCl}_{3}\right.$, 293K, TMS): 7.01-7.06 (1H, m), 7.15-7.19 (2H, m), 7.28-7.47 (10H, m), 7.53-7.57 $(4 \mathrm{H}, \mathrm{m}) ;{ }^{13} \mathrm{C}$ NMR $\left(75 \mathrm{MHz}, \mathrm{CDCl}_{3}, 293 \mathrm{~K}, \mathrm{TMS}\right)$ : Complex peak patterns due to fluorine and phosphorous coupling; 118.59, 122.20, 125.14, 125.18, 125.23, $125.27,125.32,125.36,125.48,125.55,125.81,127.55,127.63,127.81,127.84$, $129.42,129.59,129.65,129.78,129.80,130.17,130.41,130.48,130.60,131.03$, 131.46, 133.67, 133.83, 134.04, 134.10, 134.22, 134.30, 134.39, 134.47, 141.19, 141.28, 141.92, 142.13, 148.50, 148.89; ${ }^{19} \mathrm{~F}$ NMR (282.2MHz, $\mathrm{CDCl}_{3}, 293 \mathrm{~K}$, TFA): $12.86 ;{ }^{31} \mathrm{P}$ NMR (121.4MHz, $\left.\mathrm{CDCl}_{3}, 293 \mathrm{~K}, \mathrm{HMPA}\right):-13.50 ;$ HRMS calculated for $\mathrm{C}_{26} \mathrm{H}_{17} \mathrm{PF}_{6}(\mathrm{M}+)$ 474.0972; Found : 474.0943; MP: $137-138^{\circ} \mathrm{C}$ (Hex) 


\section{Intramolecular Kinetic Isotope Effect Experiment}<smiles>[2H]c1ccccc1OCc1ccccc1Br</smiles>

$$
\underset{\mathrm{K}_{2} \mathrm{CO}_{3}, \mathrm{DMA}}{\stackrel{\mathrm{Pd}(\mathrm{OAC})_{2}}{2 \text { Ligand }}}
$$<smiles>[2H]c1cccc2c1OCc1ccccc1-2</smiles><smiles>c1ccc2c(c1)COc1ccccc1-2</smiles>

$\mathrm{k}_{\mathrm{H}} / \mathrm{k}_{\mathrm{D}}=\mathbf{3 . 5}$

\section{(2-bromobenzyloxy)-2-d-benzene (A)}

$\mathrm{R}_{\mathrm{f}}=0.64$ on silica gel (5\% EtOAc:Hexanes); IR $\left(v_{\max } / \mathrm{cm}^{-1}\right) 3030$ (weak), 1515, 1240, 1028, 819, 735 ; ${ }^{1} \mathrm{H}$ NMR $\left(300 \mathrm{MHz}, \mathrm{CDCl}_{3}, 293 \mathrm{~K}, \mathrm{TMS}\right): 5.14(2 \mathrm{H}, \mathrm{s}), 6.98$ $(2 \mathrm{H}, \mathrm{m}), 7.18(1 \mathrm{H}, \mathrm{td}, J=7.6$ et $J=1.6), 7.31(3 \mathrm{H}, \mathrm{m}), 7.57(2 \mathrm{H}, \mathrm{m}) ;{ }^{13} \mathrm{C} \mathrm{NMR}$ $\left(75 \mathrm{MHz}, \mathrm{CDCl}_{3}, 293 \mathrm{~K}, \mathrm{TMS}\right): 69.27,114.87,121.16,122.23,127.56,128.84$, $129.18,129.43,129.53,132.59,136.33,158.36$; HRMS calculated for $\mathrm{C}_{10} \mathrm{H}_{13} \mathrm{DBrO}(\mathrm{M}+)$ 263.0055; Found 263.0066;

Crude NMR of the experiment shown at the end of experimental data.

\footnotetext{
${ }^{1}$ Bowman, R., Mann, E., Parr, J., J. Chem. Soc., Perkin Trans. 1, 2000, 2991-2999

${ }^{2}$ Rossi, R., Carpita, A., Pazzi, P., Mannina, L., Valensin, D. Tetrahedron, 1999, 55, 11343-11364

${ }^{3}$ Ames, D.E.., Opalko, A., Tetrahedron, 1984, 40, 1919-1925

${ }^{4}$ Parham, W.E., Jones, L.D., Sayed, Y.A., J. Org. Chem. 1976, 41,1184

${ }^{5}$ Harayama, T., Akiyama, T.,Akamatsu, H., Kazuko., Abe, H., Takeuchi, Y., Synthesis, 2001, 3, 444-450

${ }^{6}$ Cas No : 776-35-2

${ }^{7}$ 2-(Diphenylphosphino)-2'-(N,N-dimethyl-amino)byphenyl is the ligand used for all cyclization reactions
} 
마을

s5

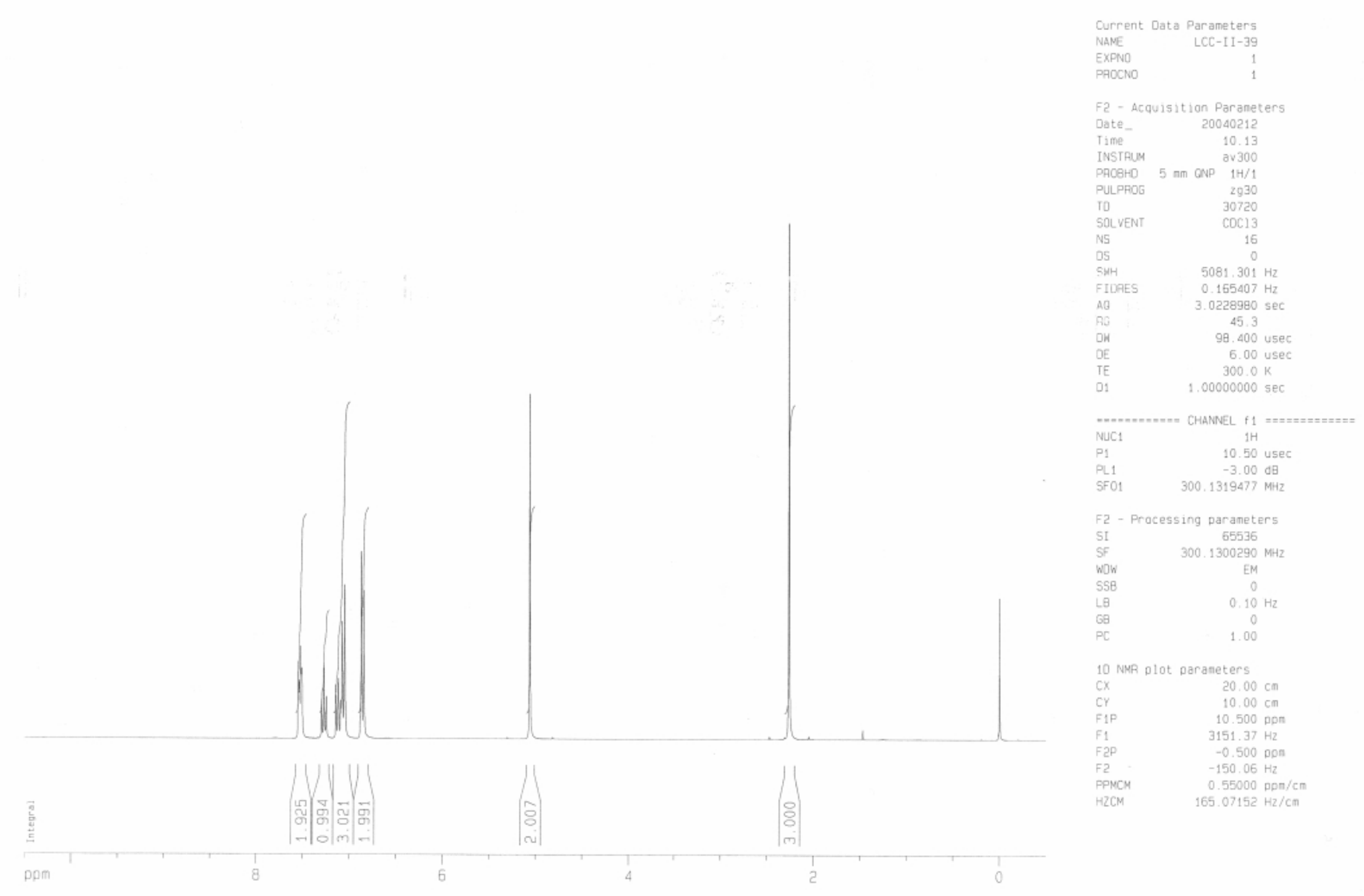


그.

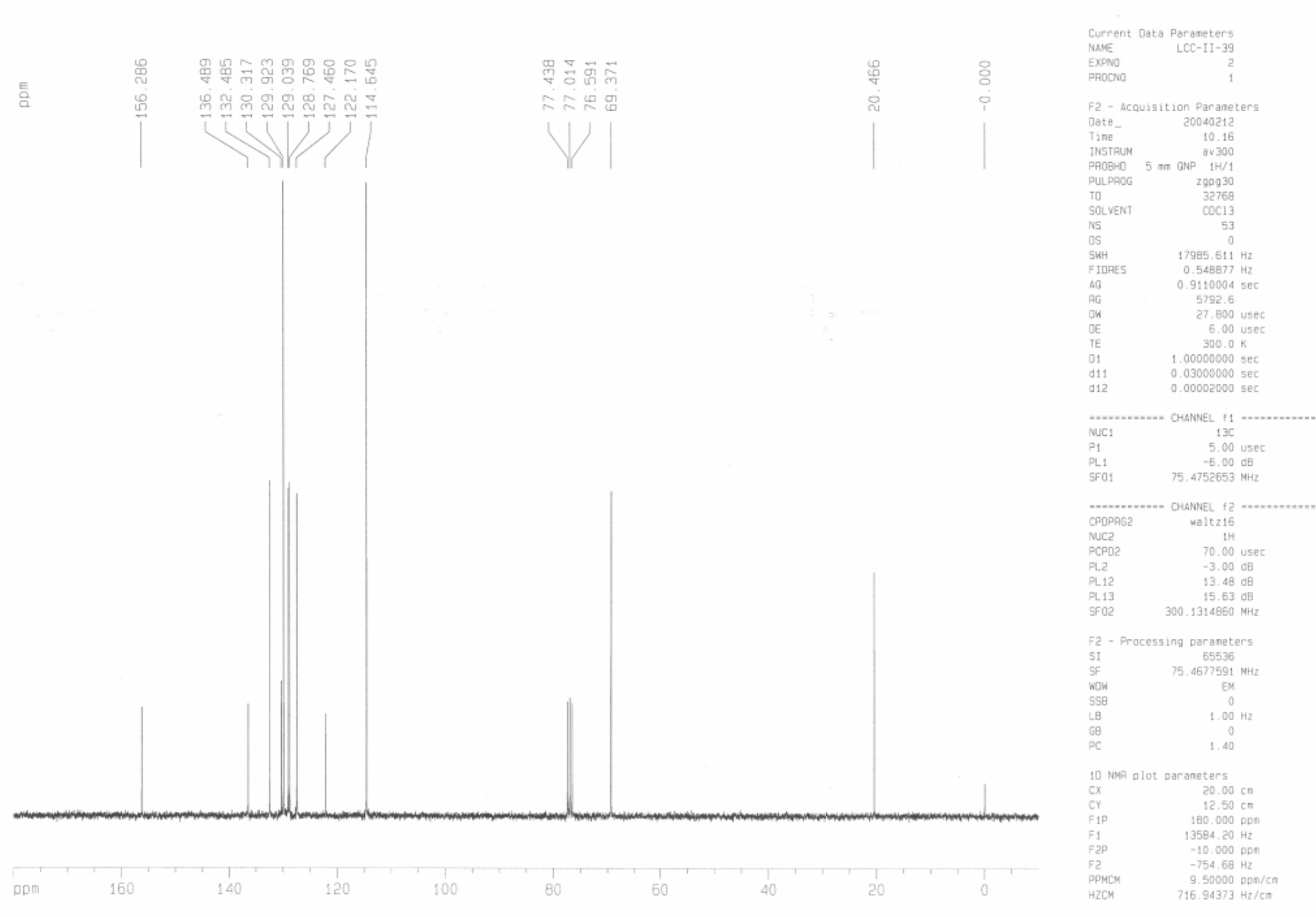



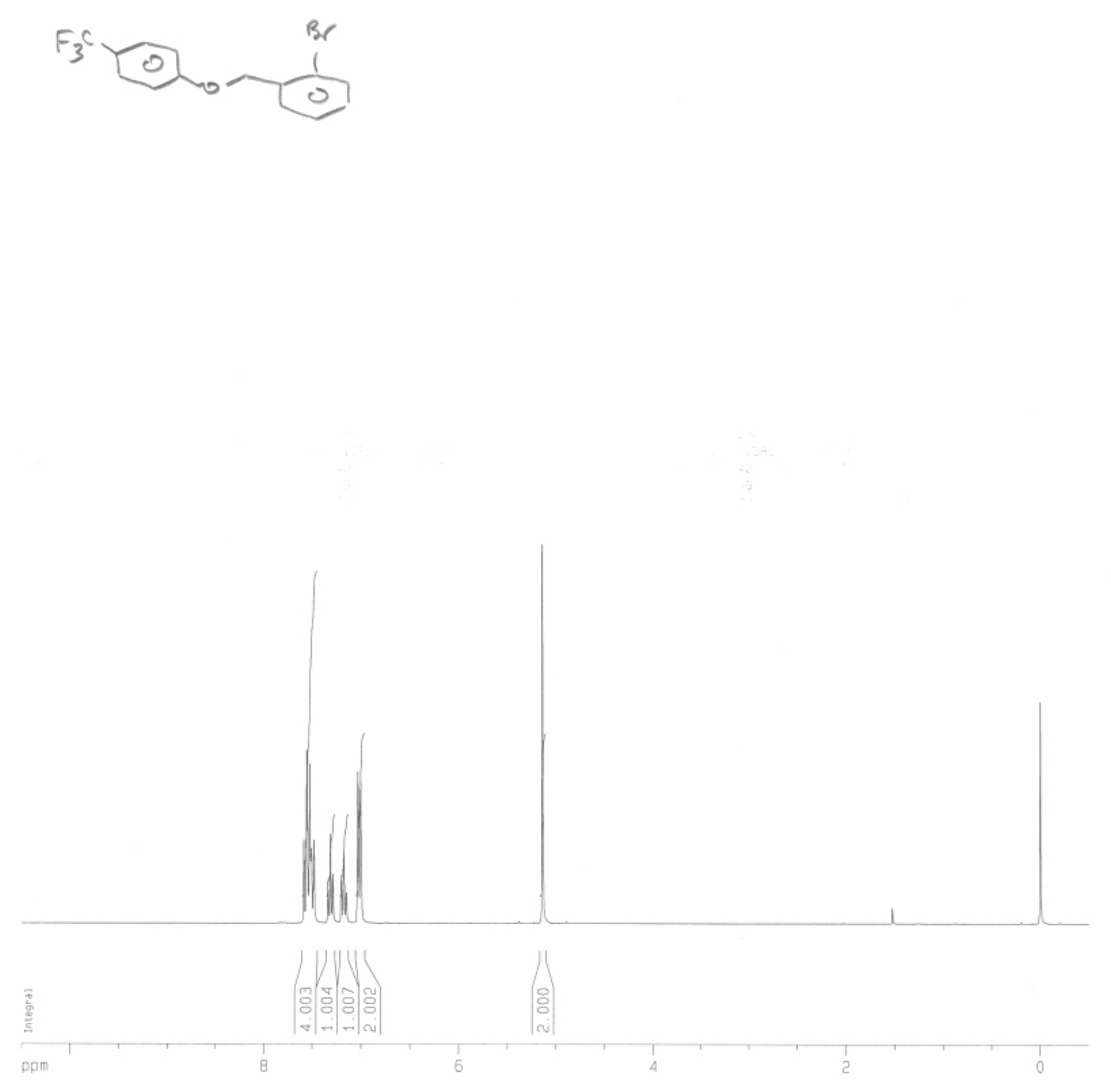

Current Data Paraneters

EXPNO
PROCNO

F2 - Acquisition Parameters

Date $\quad 20031105$

$\begin{array}{lr}\text { Time } & 13.02 \\ \text { INSTRLM } & \text { ar300 }\end{array}$

$\begin{array}{lll}\text { PAOBHO } 5 \mathrm{~mm} \text { ONP } & 1 \mathrm{H} / \\ \text { PULPAOG } & \end{array}$

$\begin{array}{lr}\text { TO } & 30720 \\ \text { SOLVENT } & \text { COC13 } \\ \text { NIS } & \text { OS }\end{array}$

$\begin{array}{lc}\text { NS } & 16 \\ \text { DS } & 0 \\ \text { SHH } & 5081.301 \mathrm{~Hz} \\ \text { FIDAES } & 0.165507 \mathrm{~Hz} \\ \text { AID } & 3.0229900 \mathrm{set}\end{array}$

$\begin{array}{lr}A Q & 3.0228980 \mathrm{sec} \\ \mathrm{AG} & 71.8 \\ \mathrm{AG} & 98.400\end{array}$

$6.00 \mathrm{usec}$
$300.0 \mathrm{~K}$

4.0000

NUC1

$\begin{array}{lr} & 10.50 \text { usec } \\ \text { PLI } & -3.00 \mathrm{~dB} \\ \text { FFo1 } & 300.1319477 \mathrm{MHz}\end{array}$

F2 - Processing parameters
51
55536

SF $\quad 300.1300104 \mathrm{MH}$

$\begin{array}{lc}\text { NOW } & \text { EM } \\ \text { SSB } & 0 \\ \text { LB } & 0.10 \mathrm{~Hz}\end{array}$

$\begin{array}{ll}\mathrm{LB} & 0.10 \mathrm{~Hz} \\ \mathrm{~GB} & 0 \\ \mathrm{PC} & 1.00\end{array}$

10 NMA plot paraneters

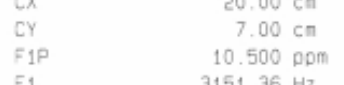

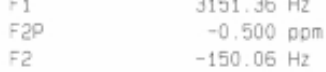

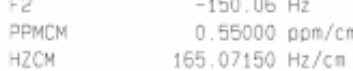


So

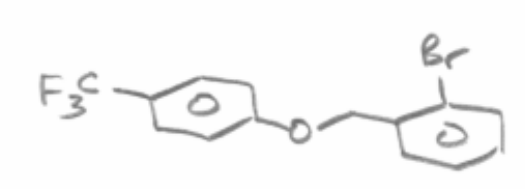

IC wi th proton decoupling
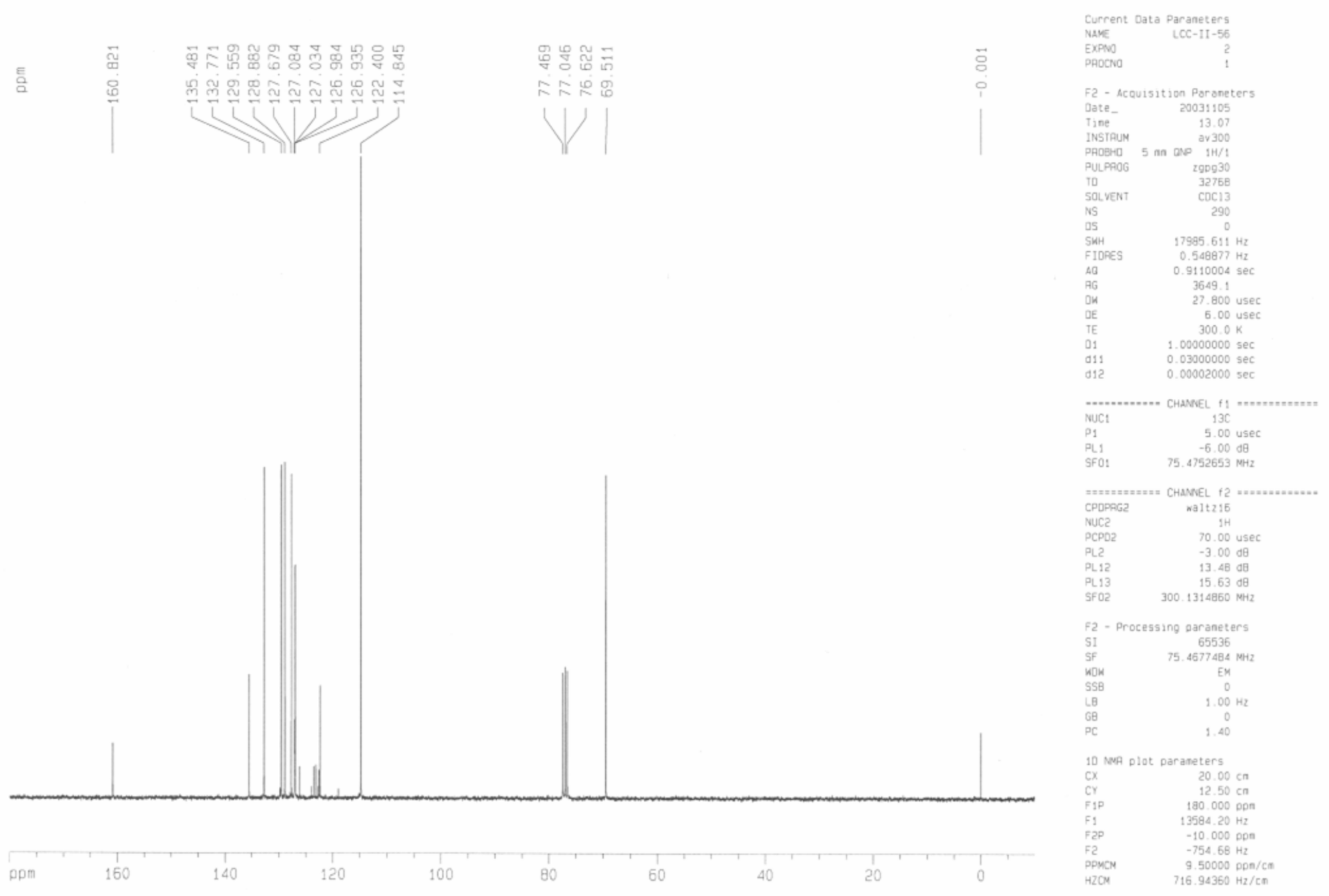

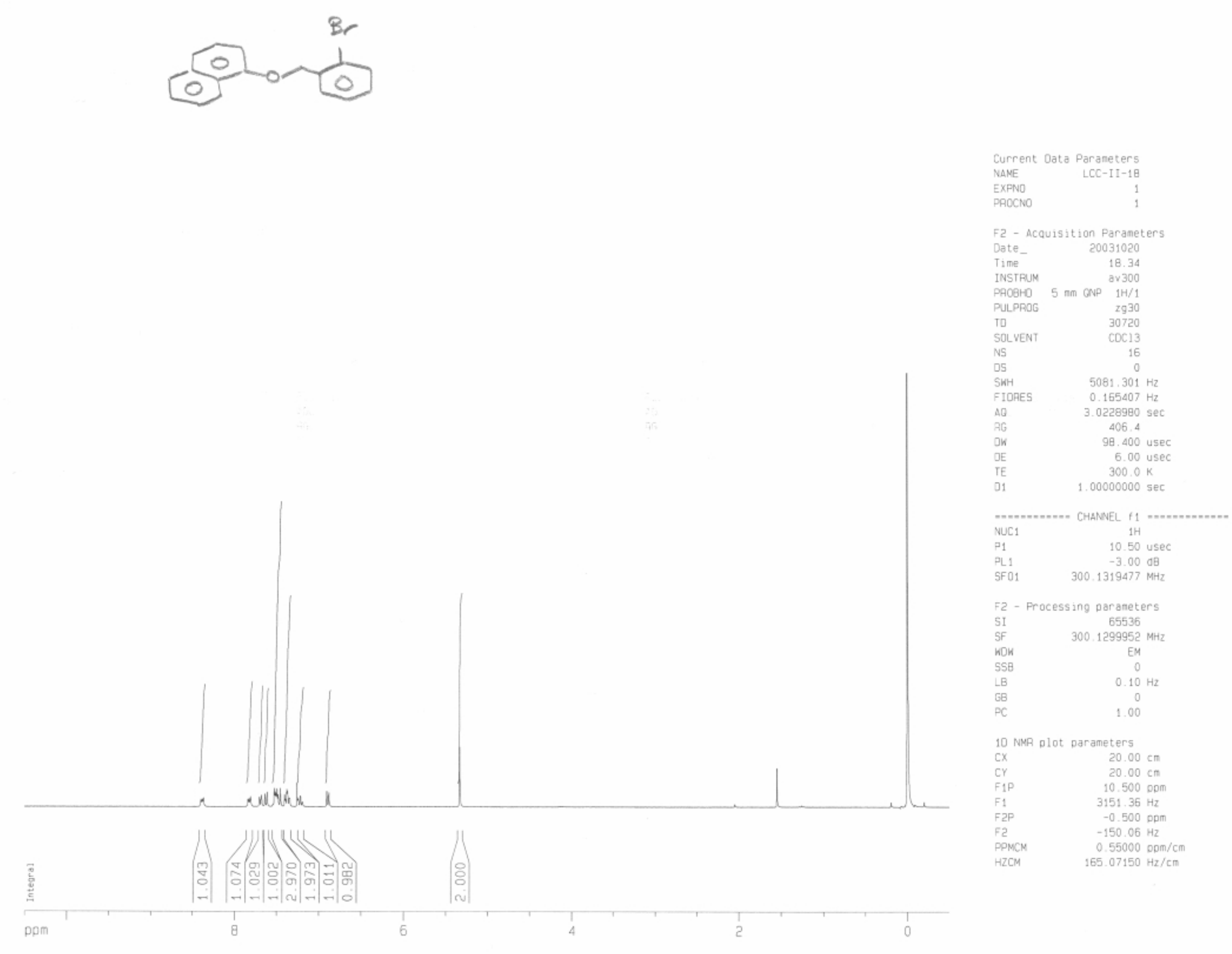


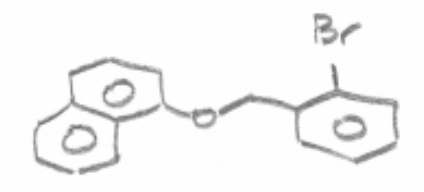

$13 \mathrm{C}$ with proton decoupling
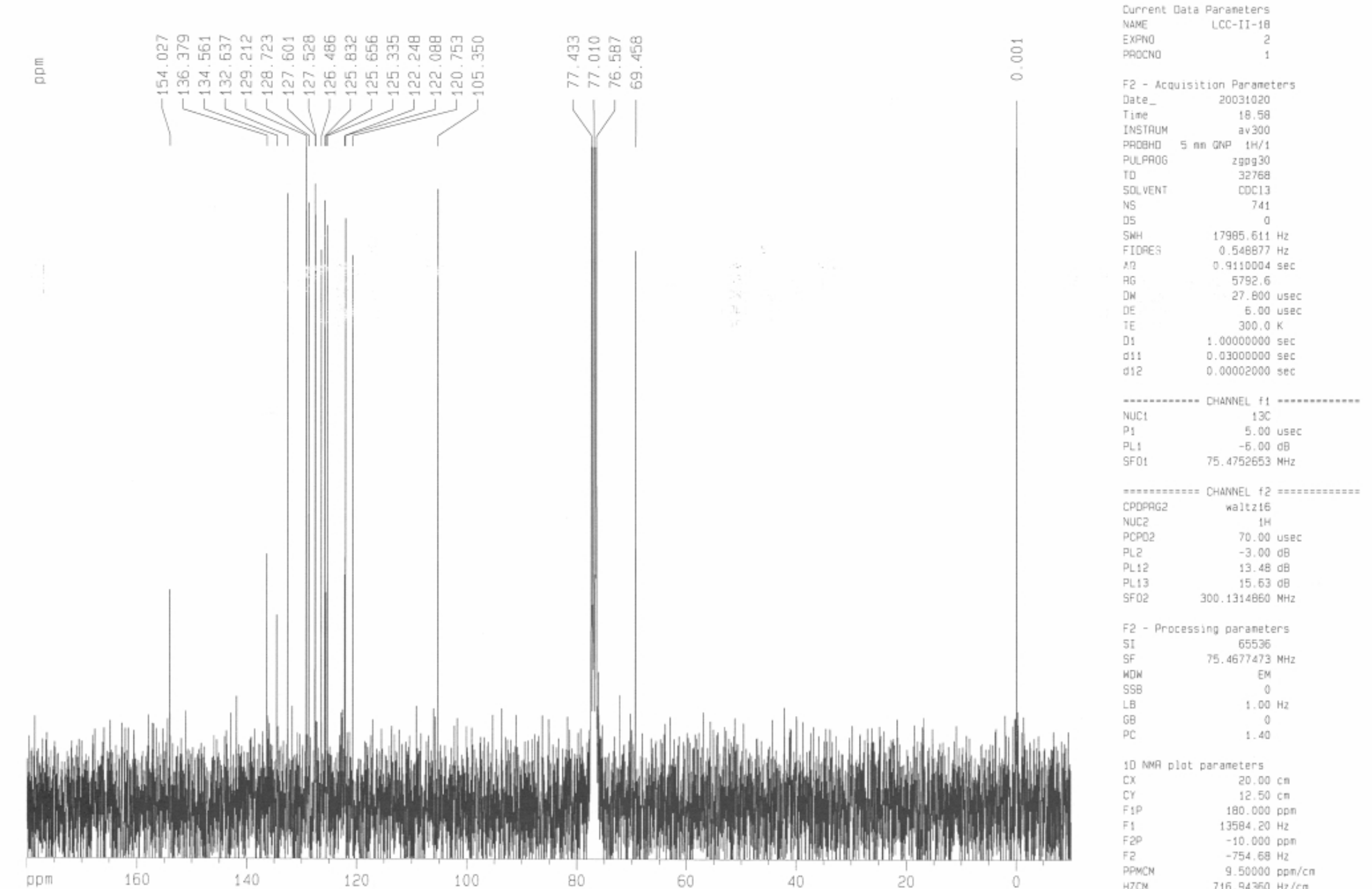

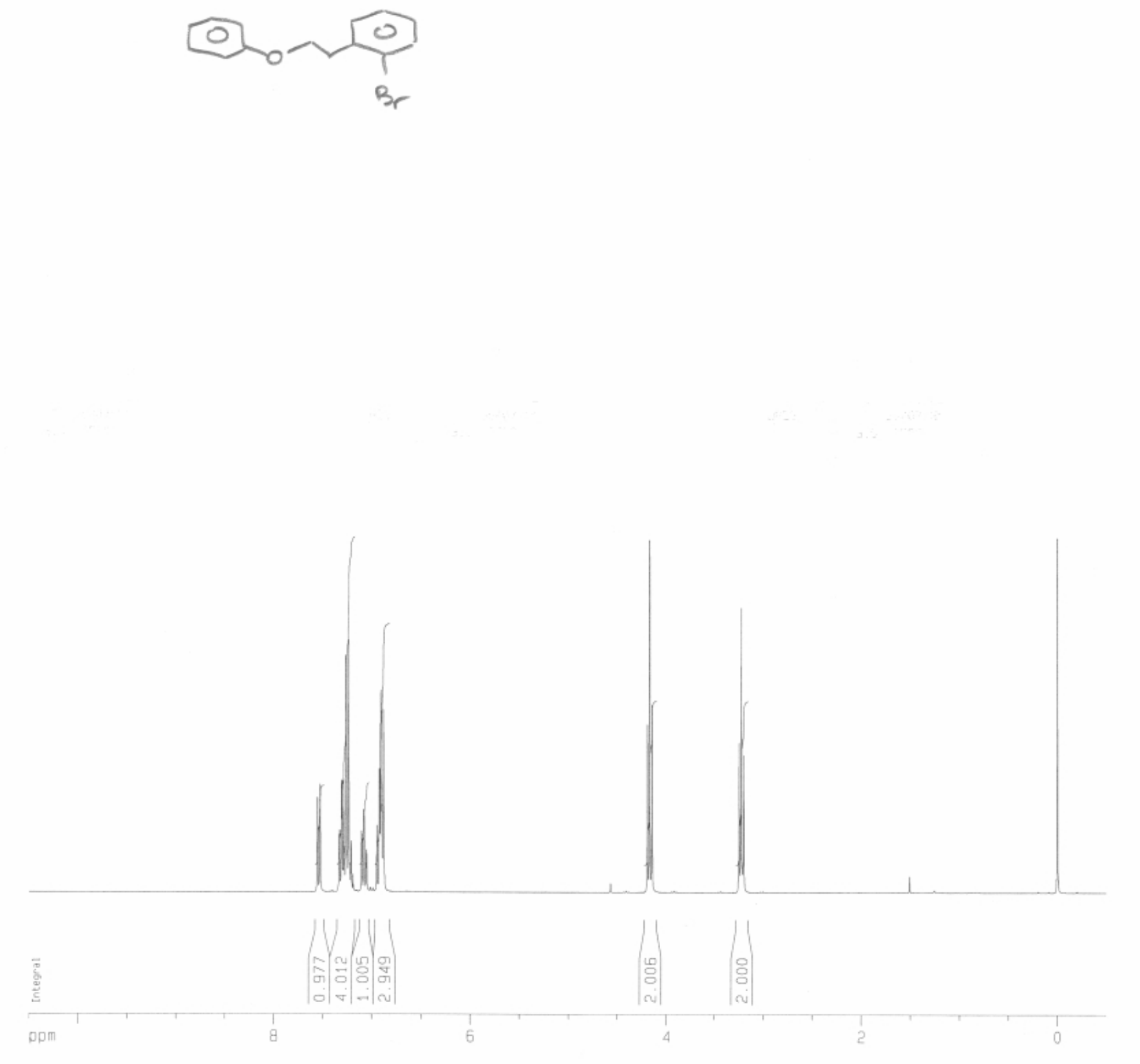

Current Data Parameters

2 - Acquisition Paraneters

bite

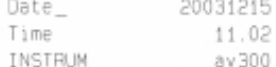

INSTFUM
PAOBHO

PULPFOG
TD

$\begin{array}{lr}3930 \\ \text { ID } & 30720 \\ \text { SOLVENT } & \text { CDC13 }\end{array}$

$\begin{array}{lc}\text { NS } & 16 \\ \text { OS } & 0 \\ \text { SWH } & 5081.301 \mathrm{~Hz} \\ \text { EIDOES } & 0.1650903\end{array}$

$50.165407 \mathrm{~Hz}$
3.0220900

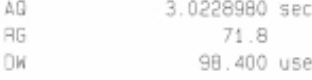

$\begin{array}{ll}\text { DE } & 6.00 \mathrm{usec} \\ \text { TE } & 300.0 \mathrm{~K}\end{array}$

z=z=sz=sz= CHANNEL $f 1$ =s:

NuC1 $\quad 10.50$ uses

PLL $\quad-3.00 \mathrm{~dB}$

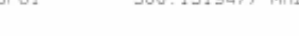

$22-$ Processing paraneters
655536

$\begin{array}{cc}\text { SI } & 65536 \\ \text { SF } & 300.1300152 \mathrm{MHz}\end{array}$

$\begin{array}{ll}\text { SF } & 300.1300152 \mathrm{MH} Z \\ \text { WDN } & \text { EM }\end{array}$

LB
GB

fu NMG plat paraneters
$20.00 \mathrm{~cm}$

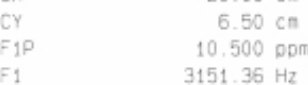

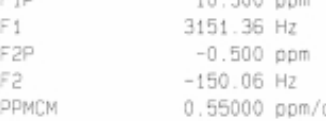

$\begin{array}{ll}\text { PPMCM } & 0.550000 \mathrm{opm} / \mathrm{cm} \\ \text { HZCM } & 165.07150 \mathrm{~Hz} / \mathrm{cm}\end{array}$ 


$$
\text { Q } 0
$$

$13 \mathrm{C}$ with proton decoupling

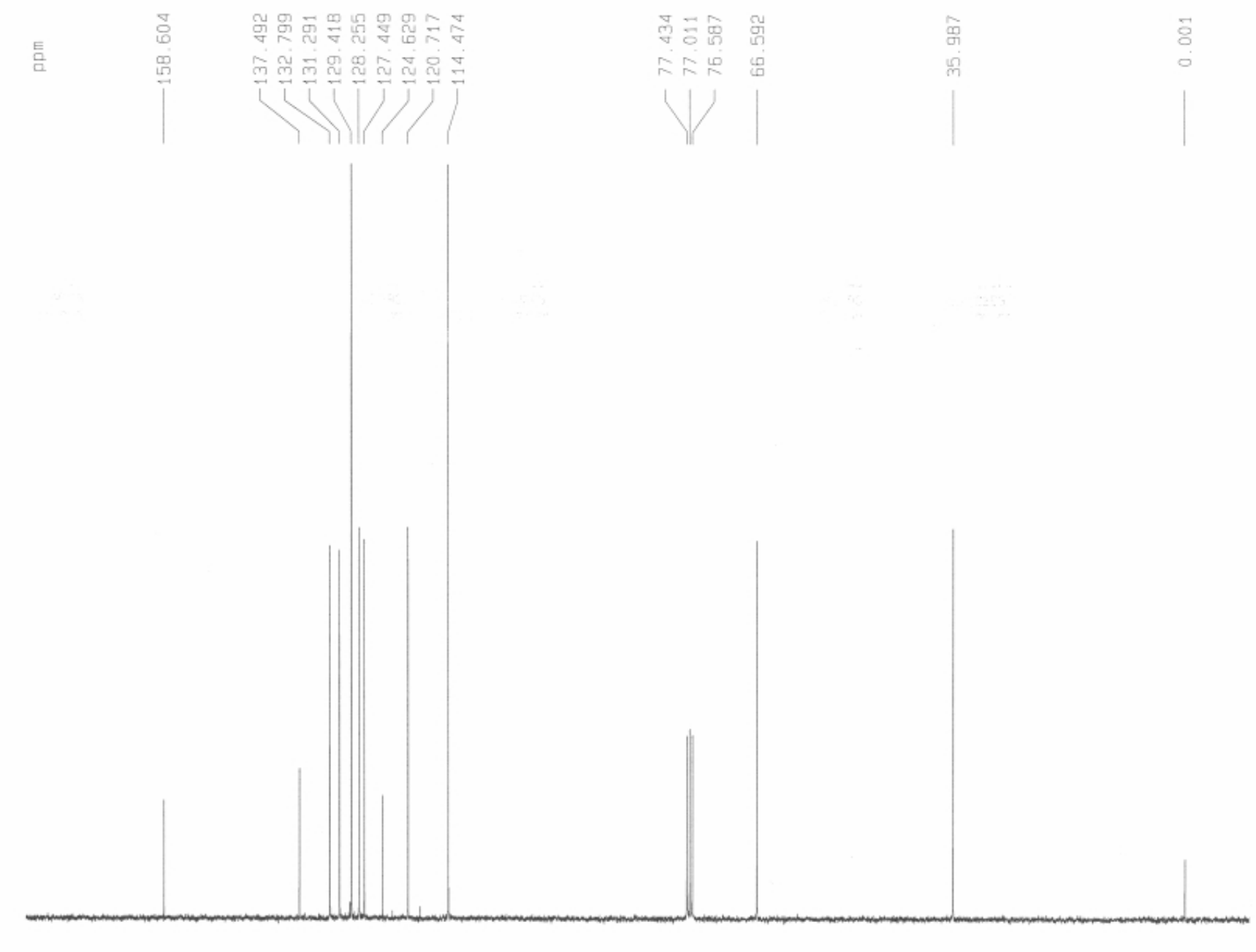

Current 0oto Pargneters
Nave
LCC7

Wuese

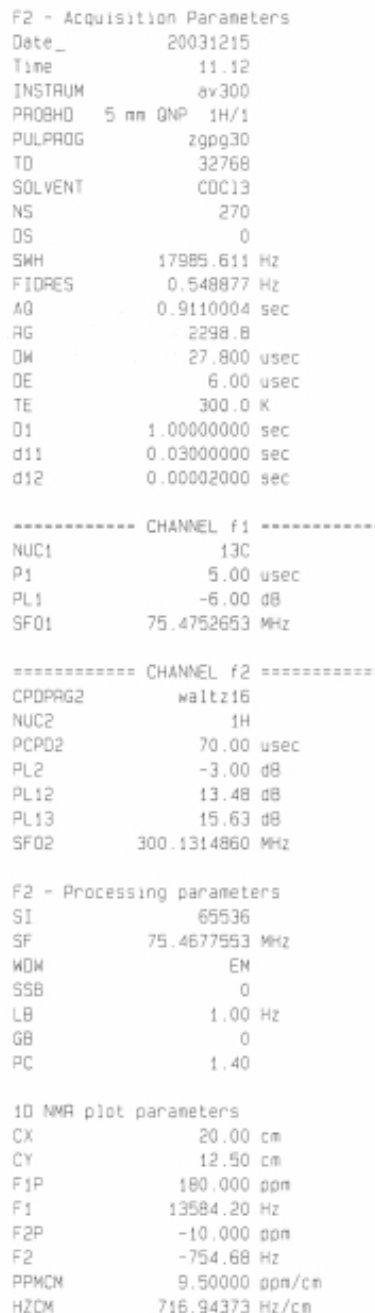


F2 - Acquisition Paraneters

Date_ 20031020

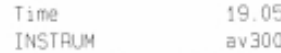

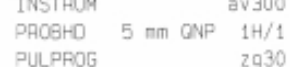

$29: 50$
TO PUPAOG
30720

$\begin{array}{lr}\text { SOLVENT } & \text { COC13 } \\ \text { NS } & 16 \\ \text { DS } & 0\end{array}$

SWH $\quad 5081.301 \mathrm{~Hz}$

AQ $\quad 3.0228980 \mathrm{sez}$

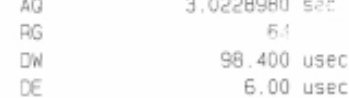

$\begin{array}{cc}D E & 6.00 \mathrm{usec} \\ T E & 300 \mathrm{~K} \\ \mathrm{TE} & 1.00000000 \mathrm{Kec}\end{array}$

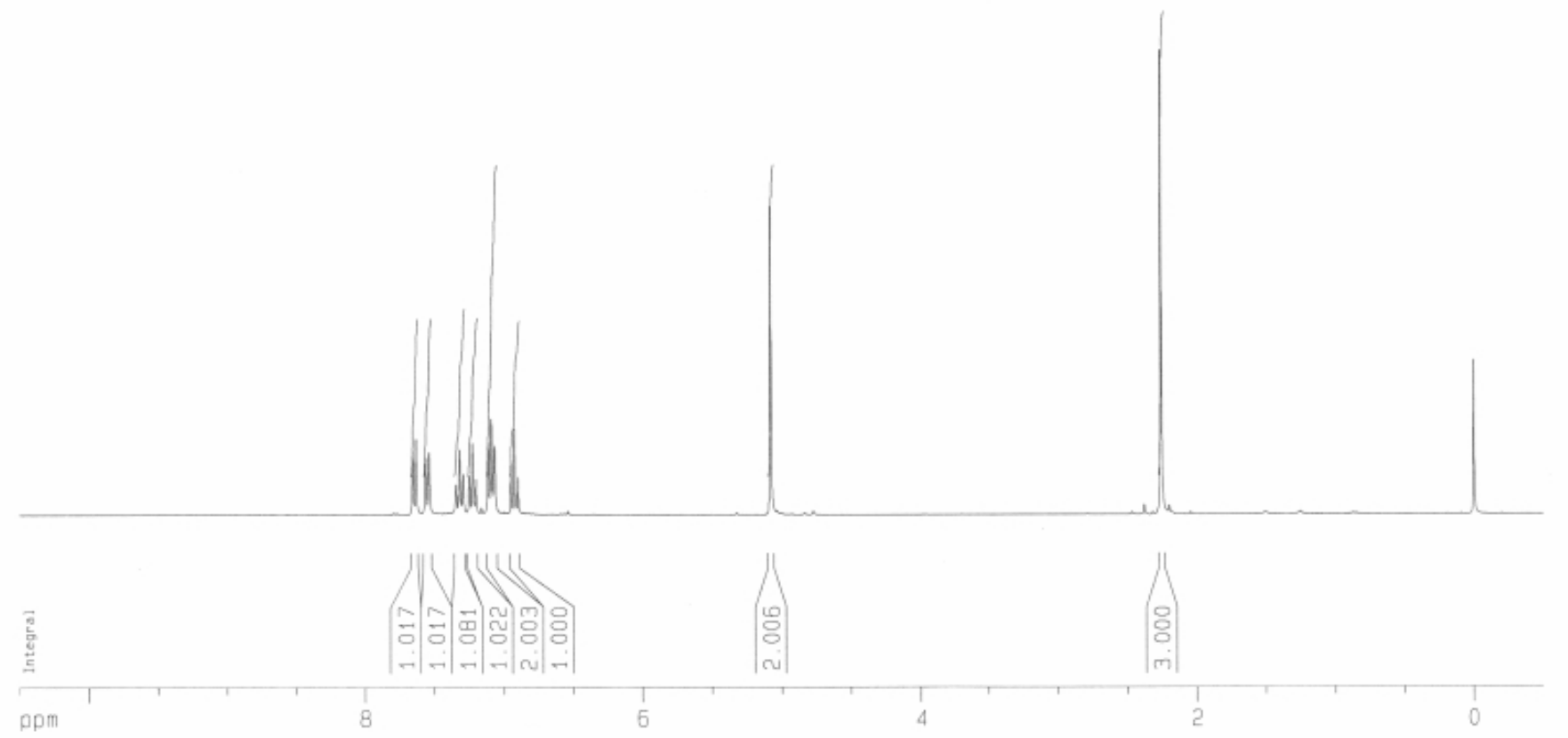

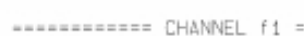

$\begin{array}{lll}\text { NuC1 } & 1 \mathrm{H} \\ \mathrm{P}_{1} & 10.50 \text { uses }\end{array}$

PL1 $\quad-3.008 \mathrm{Bg}$

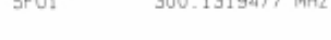

F2 - Processing paraneters

$\begin{array}{ll}\text { SI } & 65536 \\ \text { SF } & 300.1300232 \\ \text { MHZ }\end{array}$

KOH
$5 S \mathrm{~B}$

$\begin{array}{lc}L B & 0.10 \mathrm{~Hz} \\ G B & 0 \\ P C & 100\end{array}$

$10 \mathrm{NMR}$ plot paraneters
CX
$20.00 \mathrm{~cm}$
$6.00 \mathrm{~cm}$

$\begin{array}{lr}\mathrm{CY} & 6.00 \mathrm{~cm} \\ \mathrm{FIP} & 10.500 \mathrm{pm} \\ \mathrm{SP} & 3151.37 \mathrm{~Hz}\end{array}$

$\begin{array}{ll}\text { F2P } & -0.500 \mathrm{ppm} \\ \text { F2 } & -150.06 \mathrm{~Hz}\end{array}$

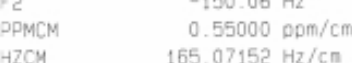




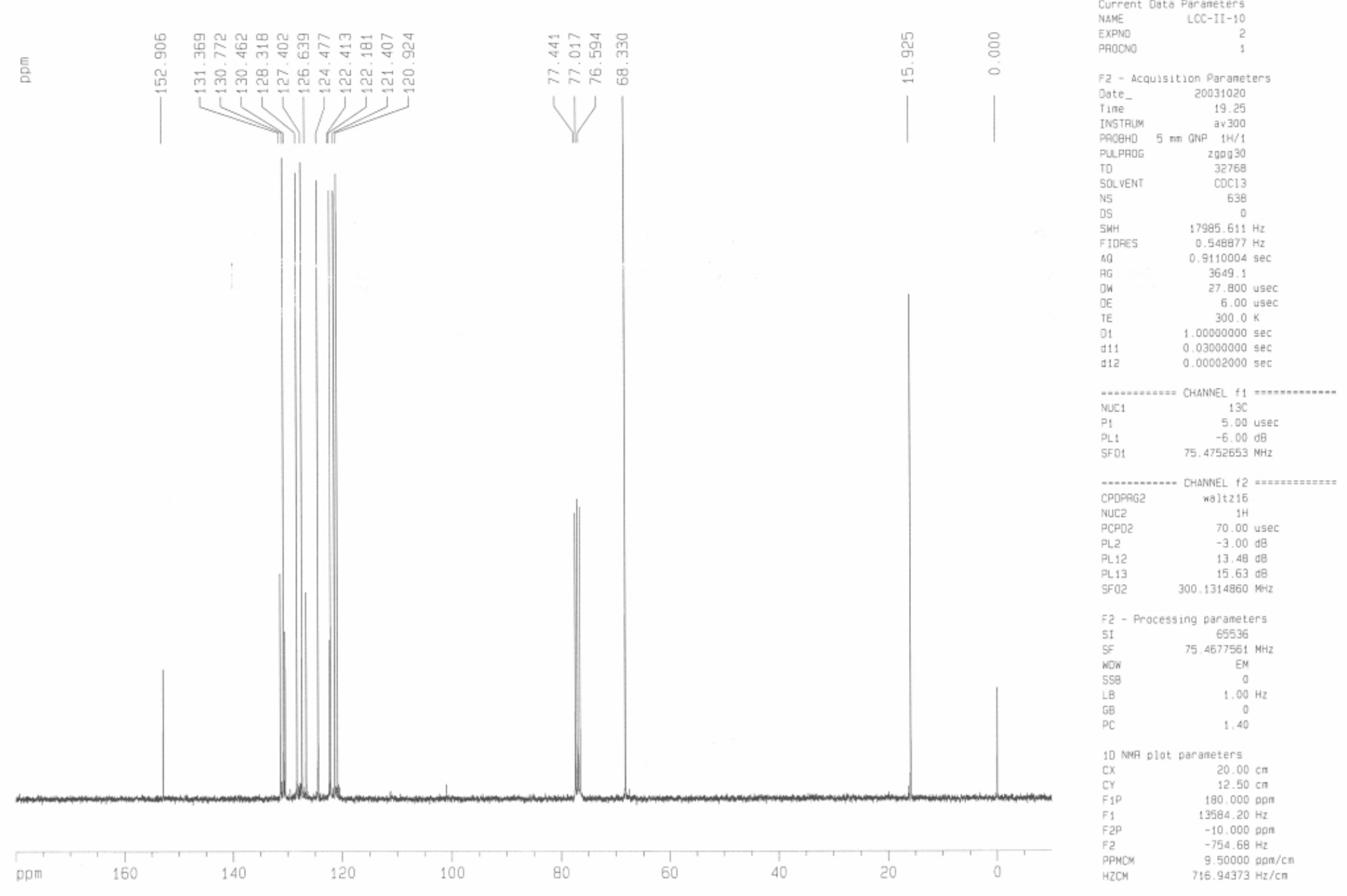



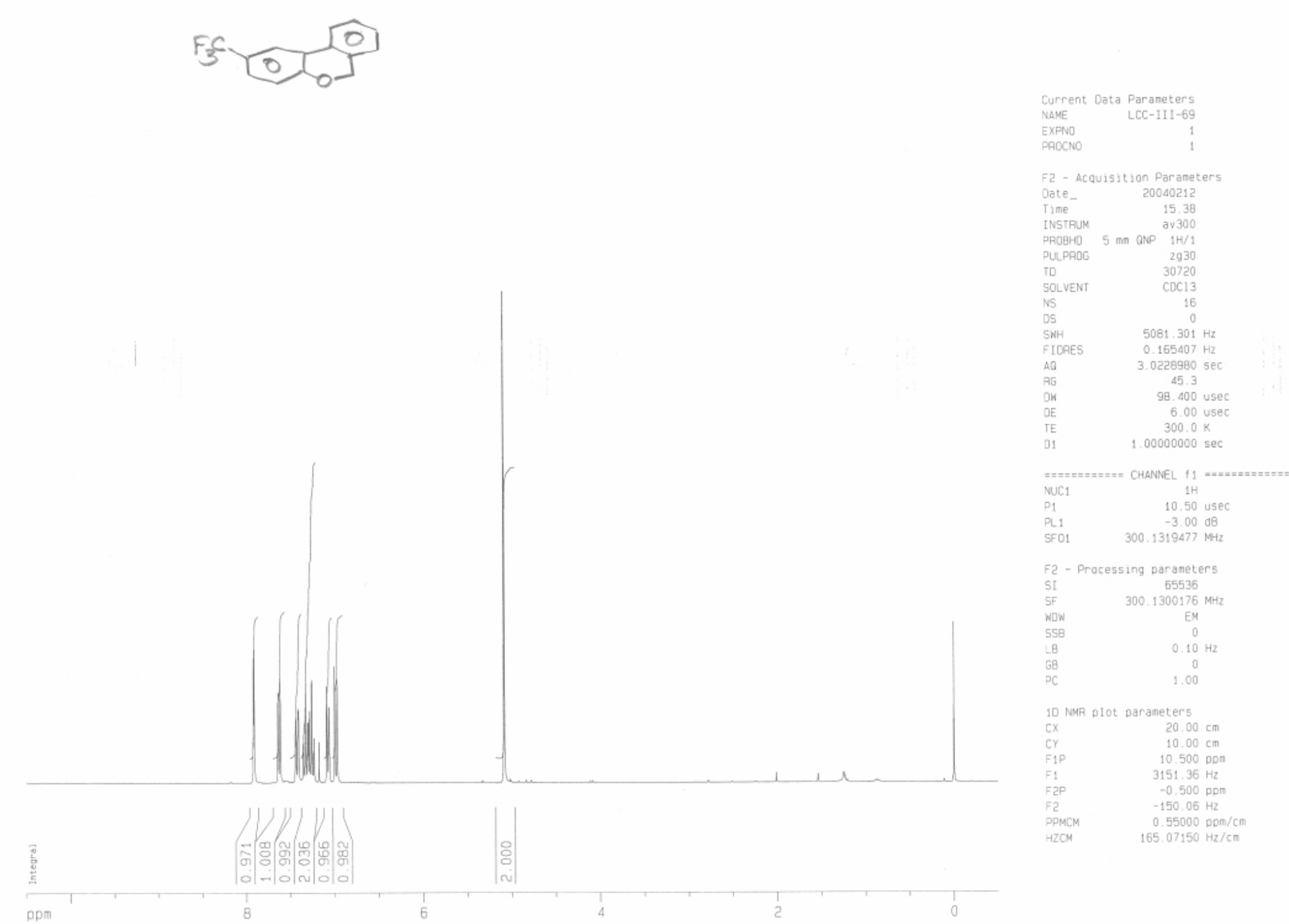
$\mathrm{F}_{3} \mathrm{Or}$

$13 \mathrm{C}$ with proton decougling
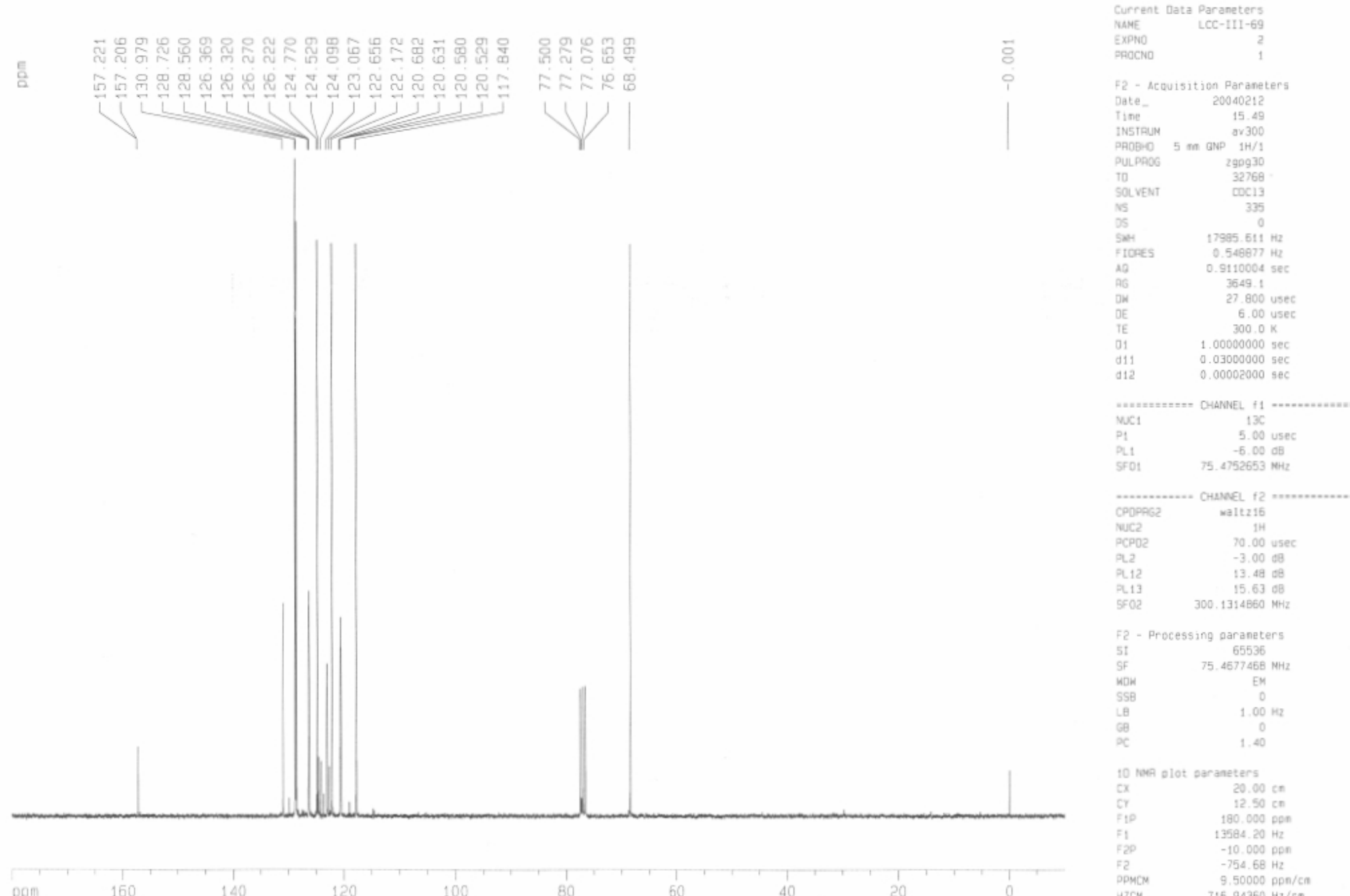


$$
\text { खिए }
$$
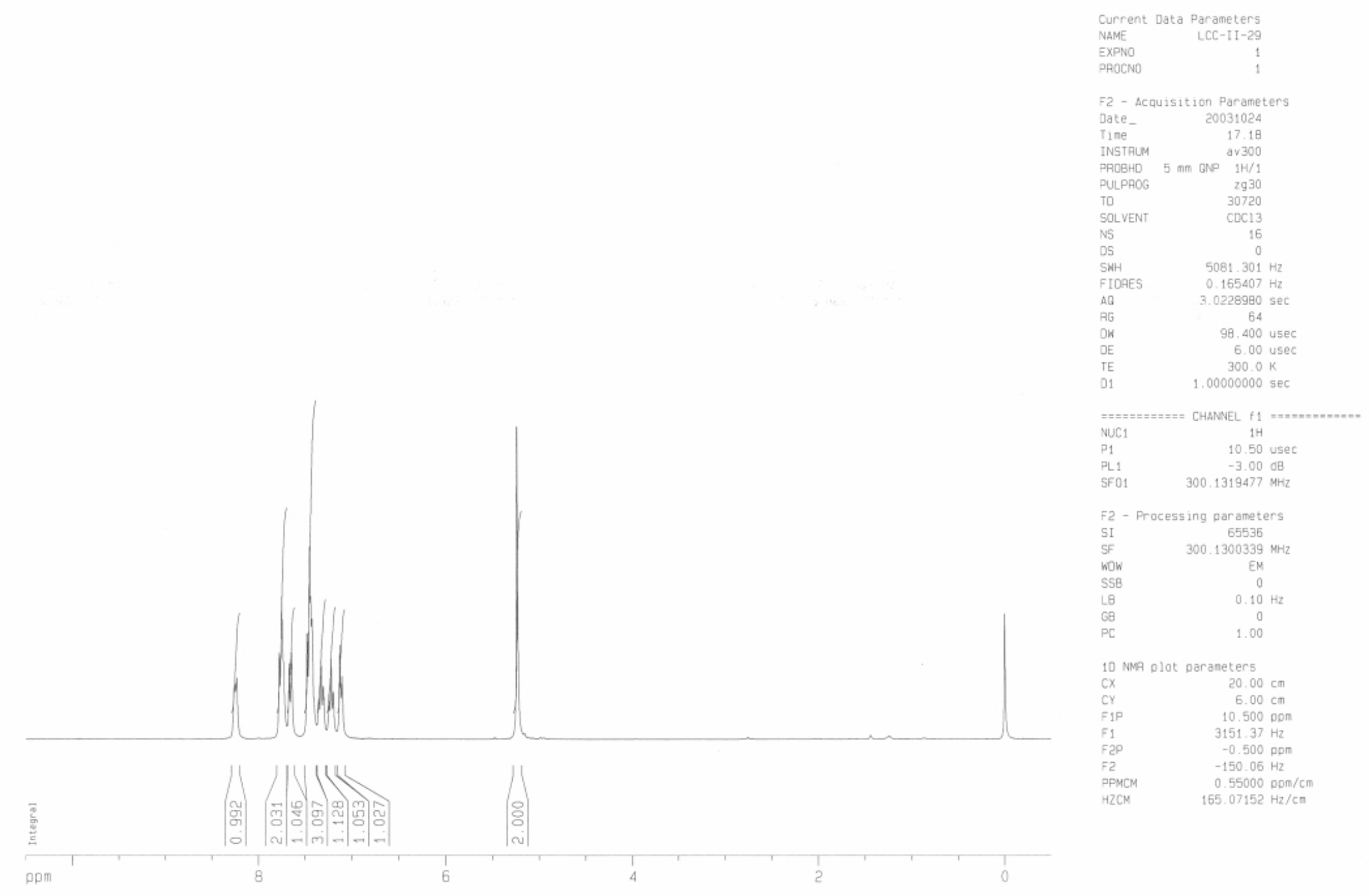


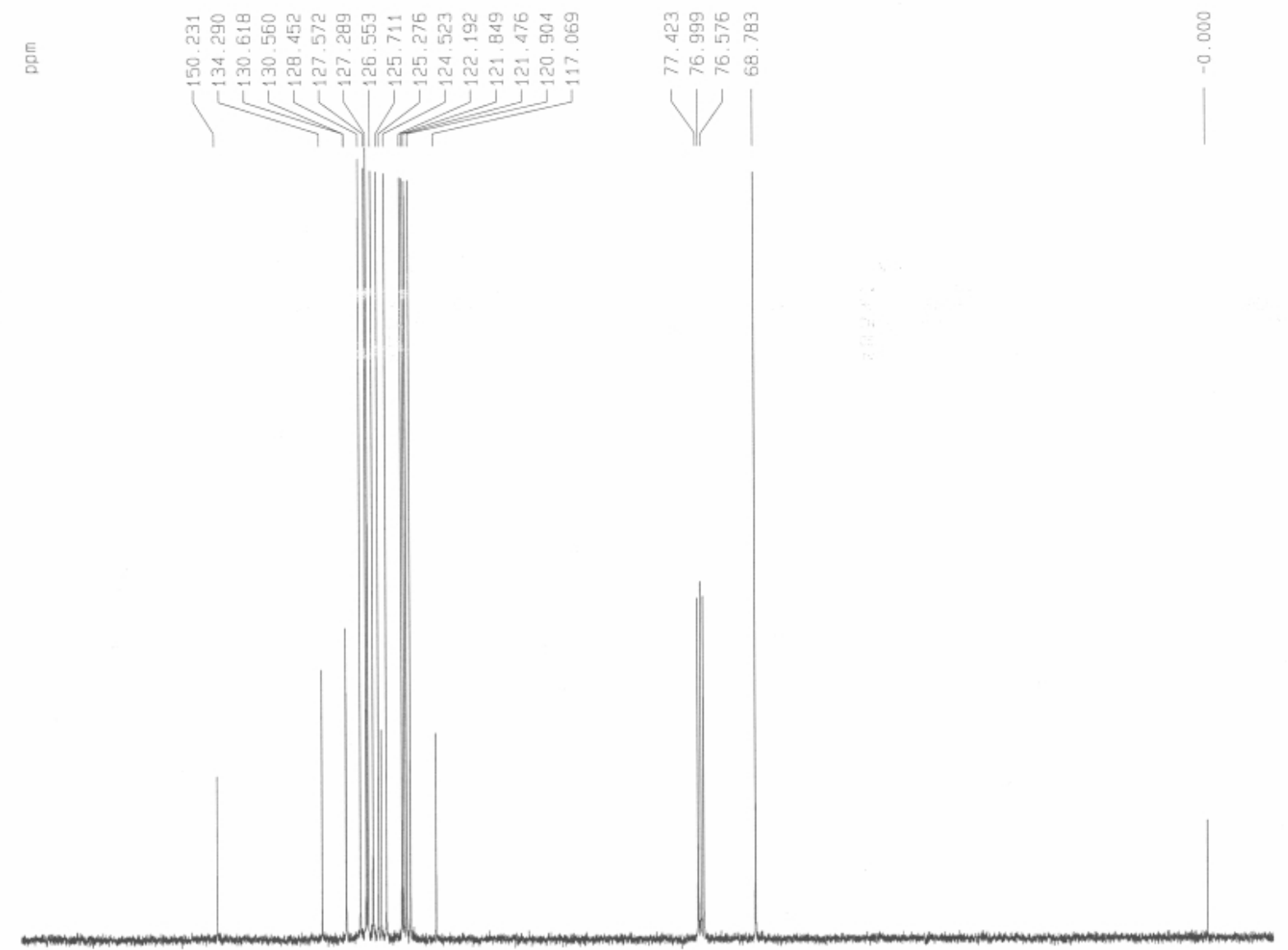

F2 - Acquisition Paraneters
Fate_ 20031024
and

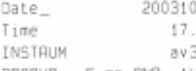

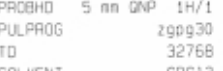

17985.011

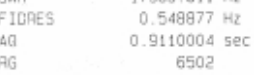

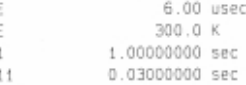

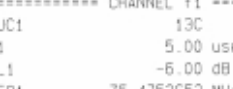

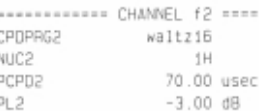

13.48
Pl.12
PL19
SF
SF

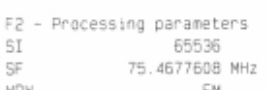

10 New plot paraneters
CX
20.00
YX
12.50

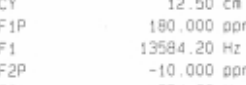



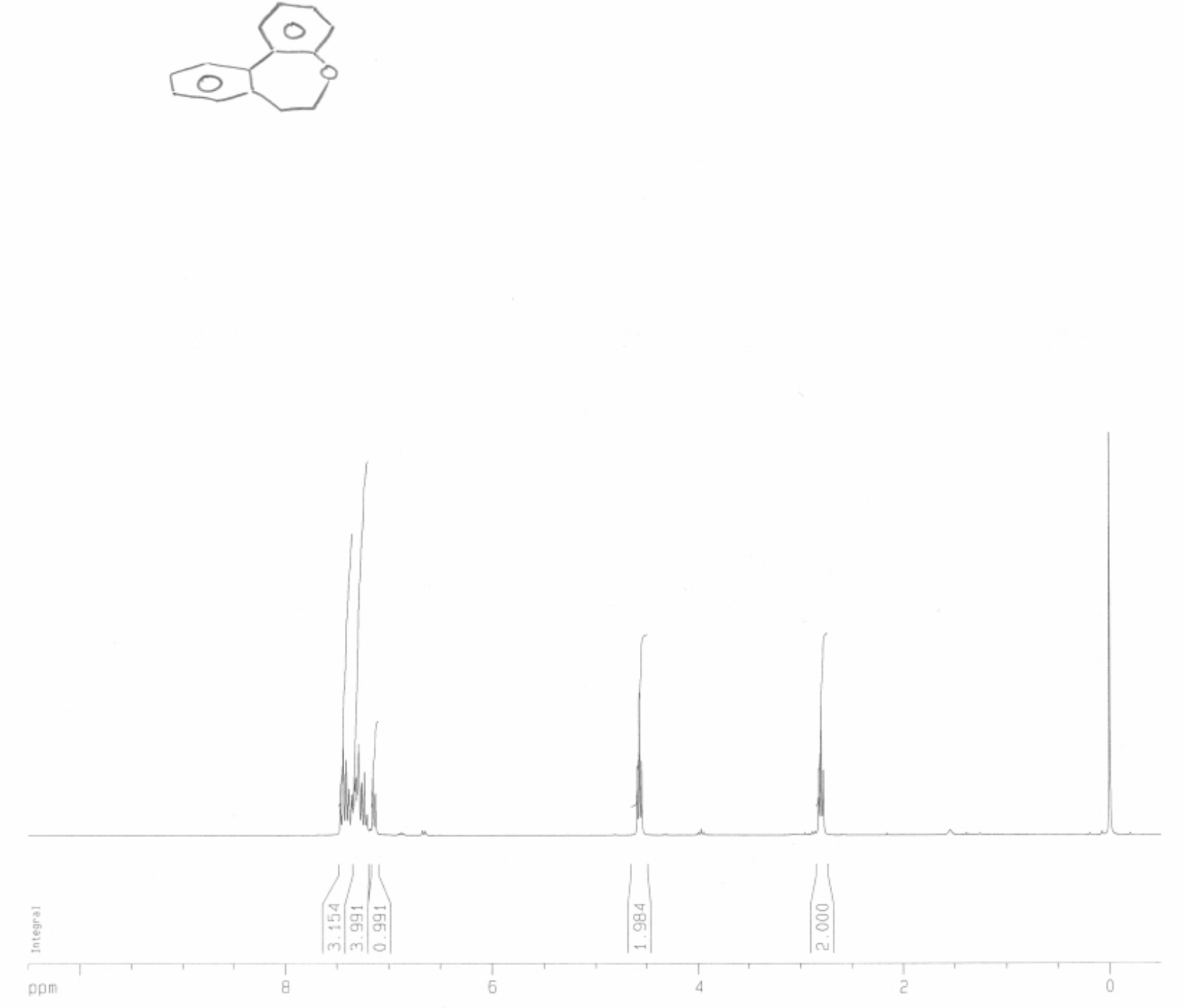

2 - Acruisition Py

$\begin{array}{ll}\text { Jate } & 20040212 \\ & 1554\end{array}$

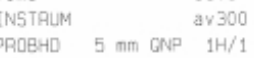

PULPAOG

\begin{tabular}{lr}
$\quad 30720$ \\
SOLVENT \\
VDC 13 \\
VI & 16 \\
\hline
\end{tabular}

SNH $\quad 5081.301 \mathrm{~Hz}$

$\begin{array}{lr}A 6 & 3.0228980 \mathrm{sec} \\ \text { OG } & 181 \\ \text { OK } & 98.400 \mathrm{usec} \\ \text { OF } & 500 \mathrm{user}\end{array}$

$300.0 \mathrm{~K}$

MC1 1 .

$\begin{array}{ll}11 & 10.50 \text { usfe } \\ \text { LL1 } & -300\end{array}$

SF01 $\quad 300.1319477 \mathrm{NH}$

F2 - Processing parameters

$\begin{array}{ll}\text { SI } & 65536 \\ \text { SF } & 300.1300019 \text { M-2 }\end{array}$

55

0.10
0

10 MMA plot parameters
CX
$20.00 \mathrm{cos}$

$\begin{array}{lr}\text { F1P } & 10.500 \mathrm{~cm} \\ \text { F1 } & 3151.36 \mathrm{~Hz}\end{array}$

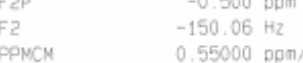

$0.55000 \mathrm{ppm} / \mathrm{cm}$
$165.07150 \mathrm{~Hz} / \mathrm{cm}$ 
$13 \mathrm{C}$ with proton decoupling
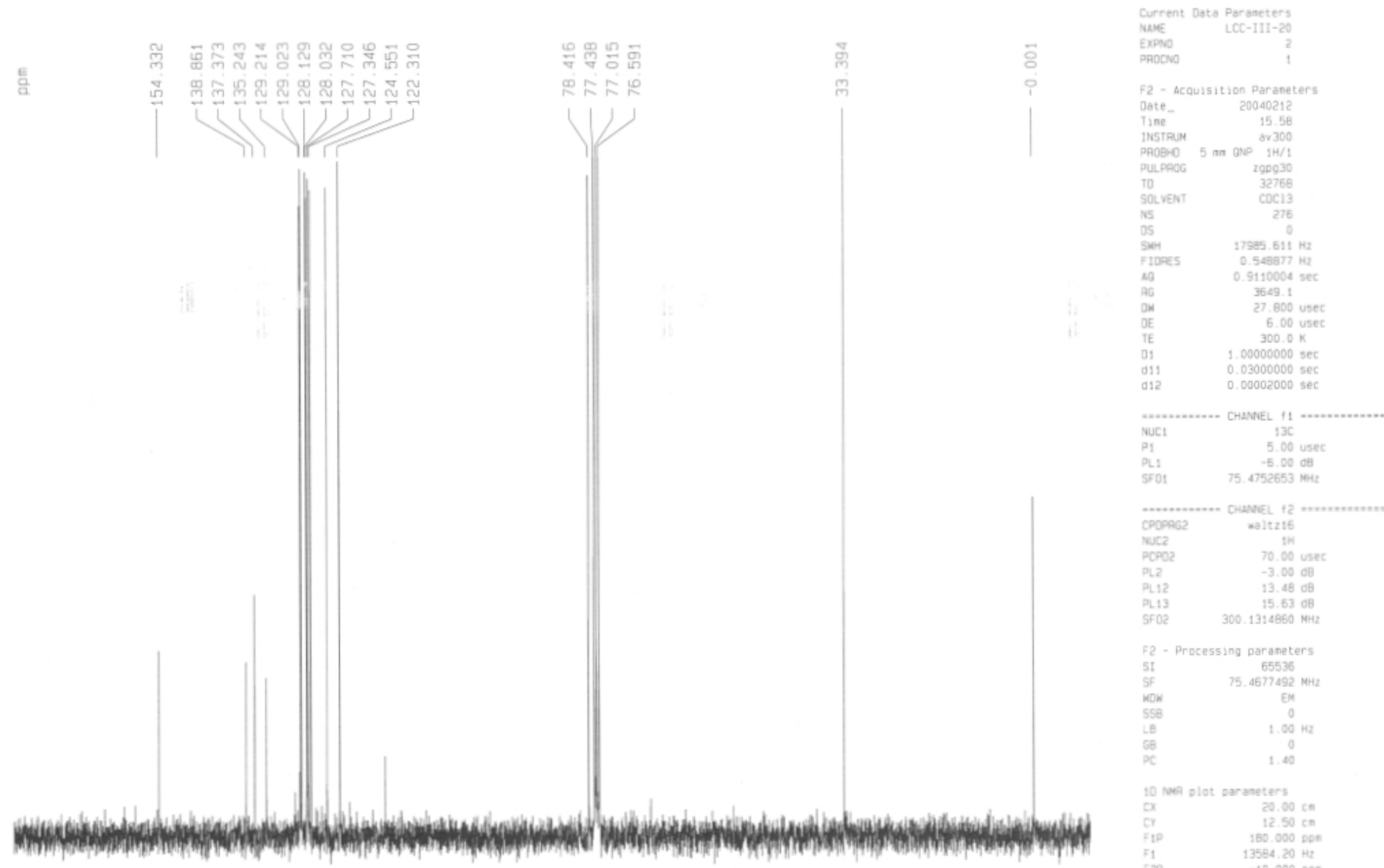

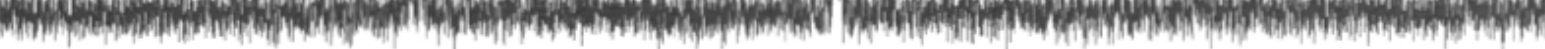

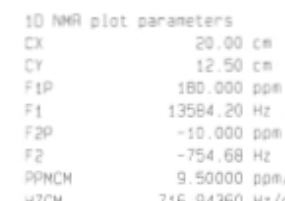


S2

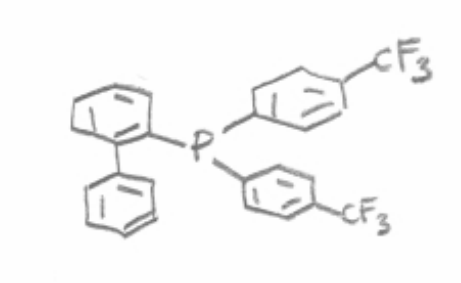

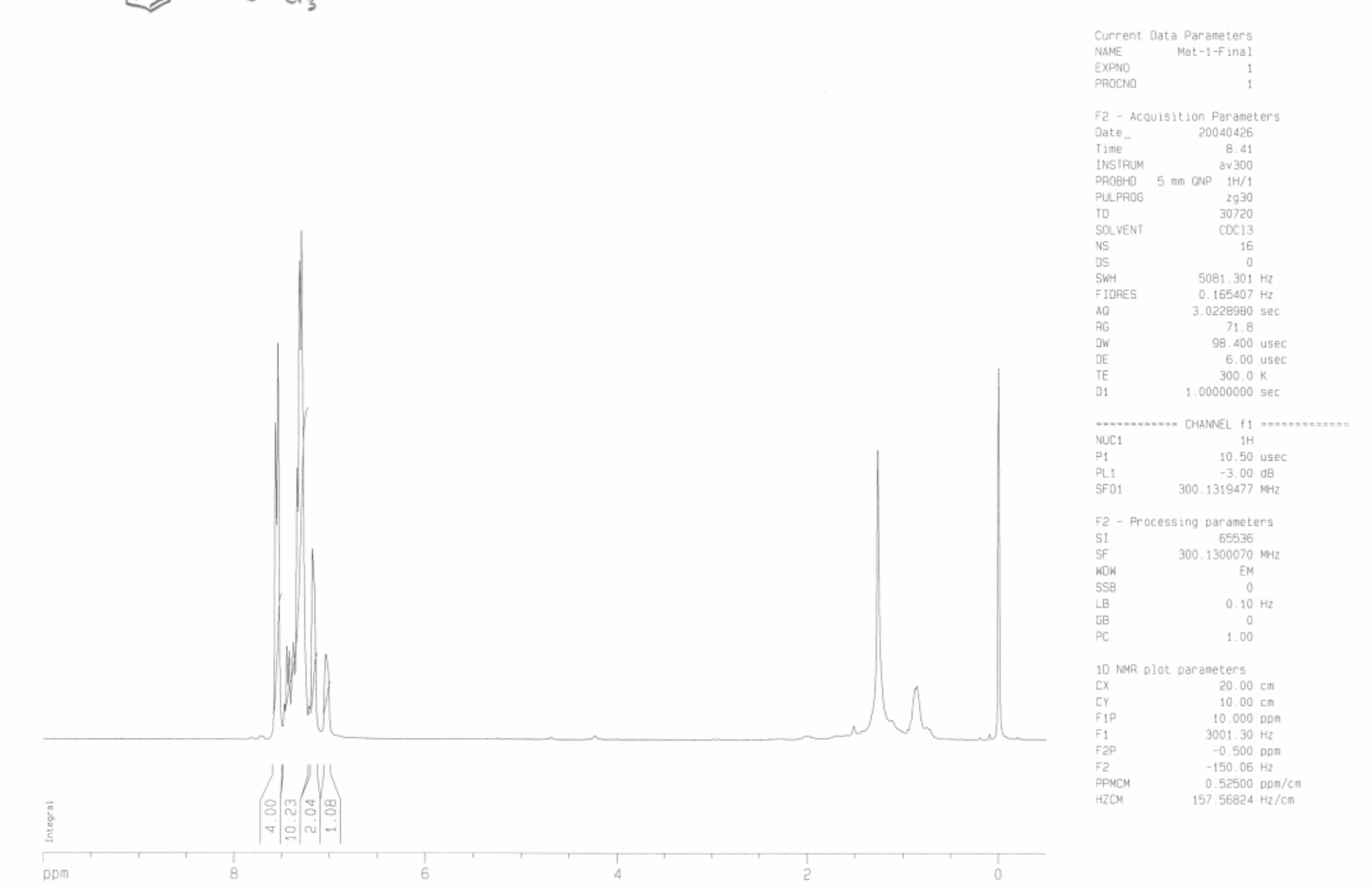




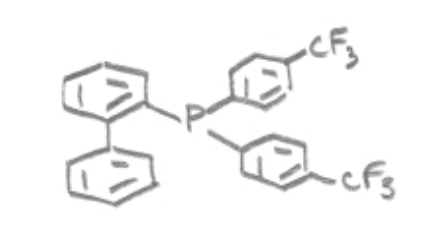

$3 \mathrm{C}$ with proton decoupling
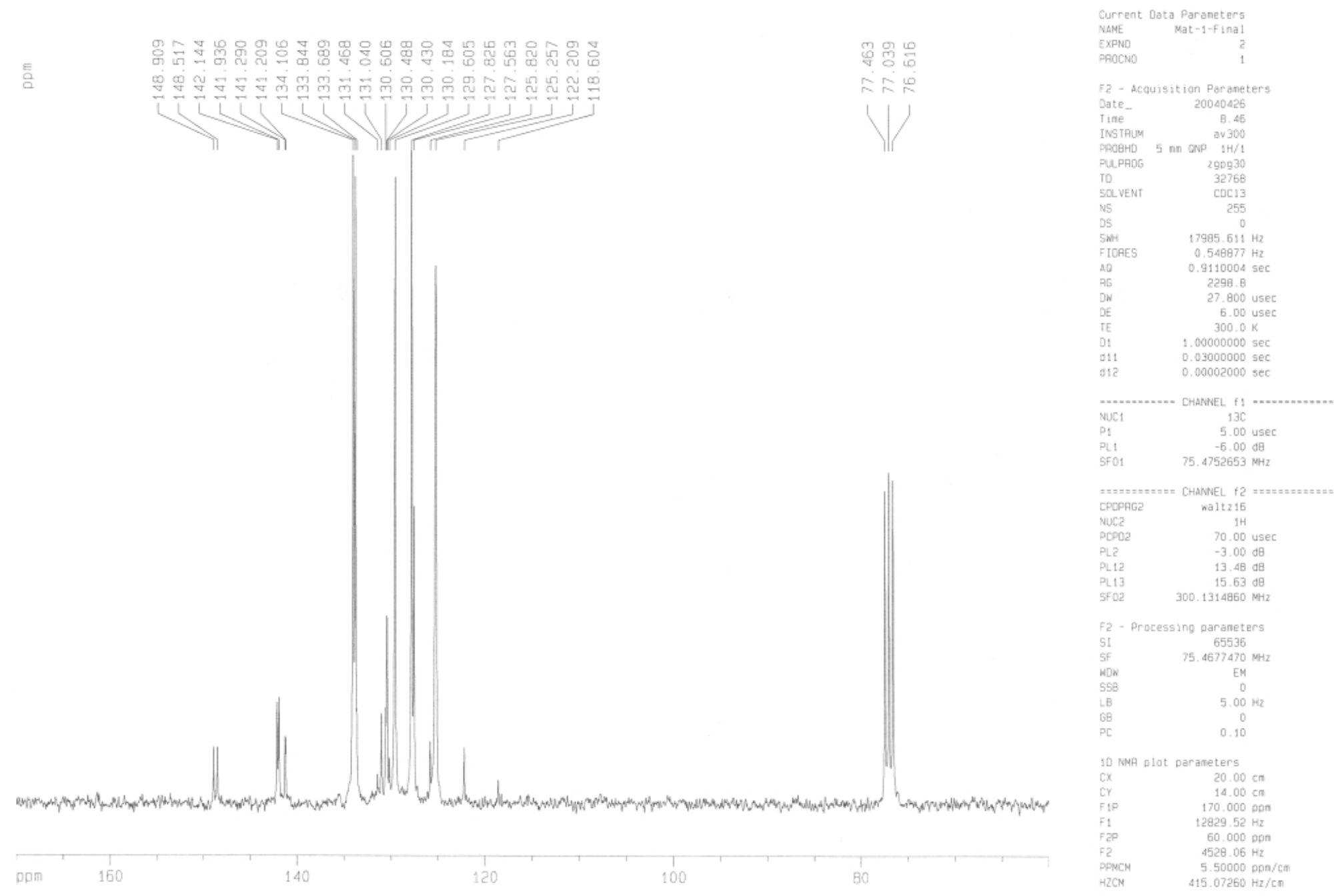

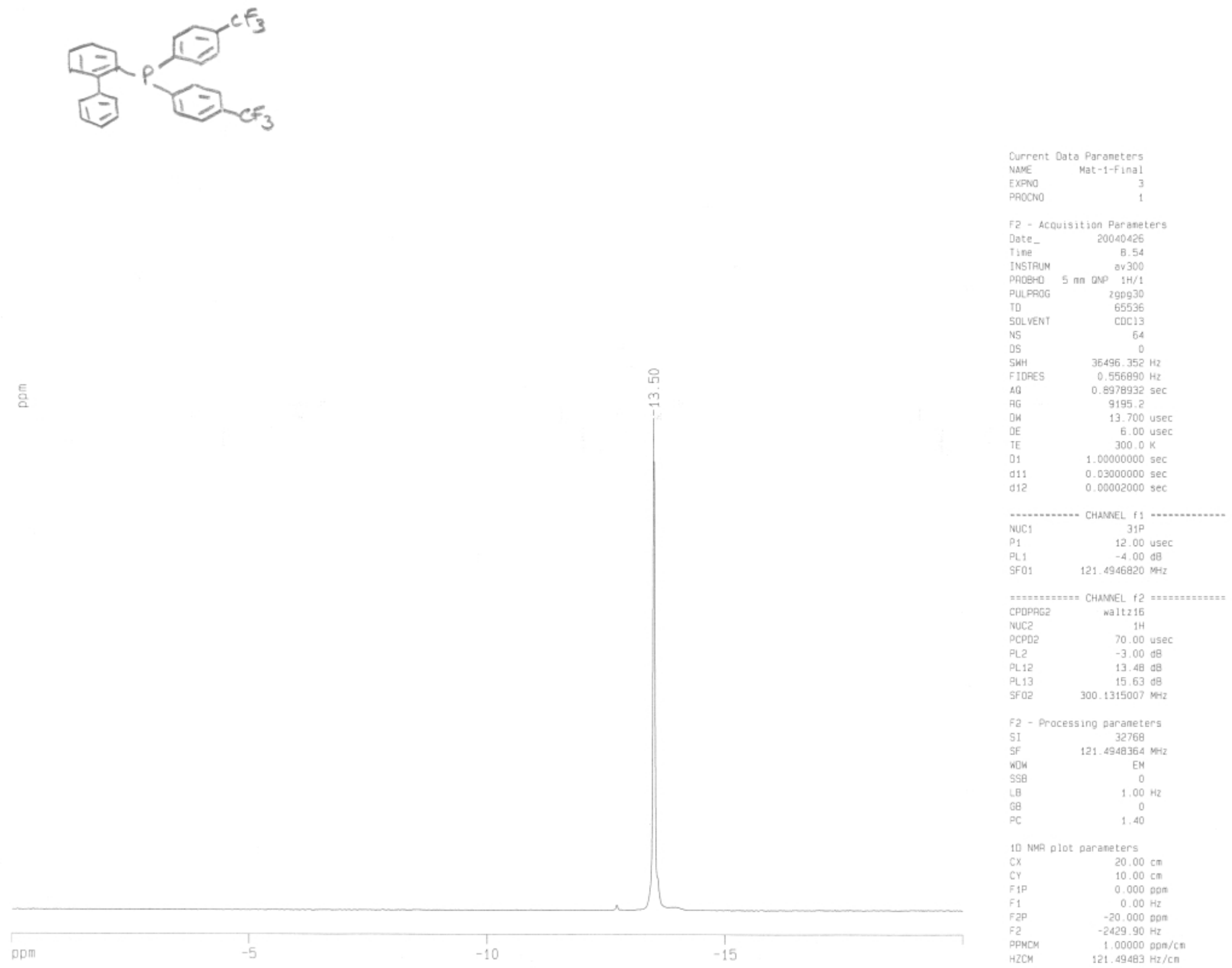


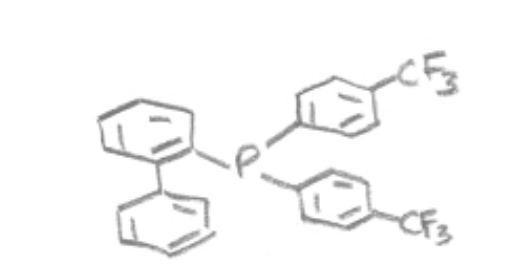


㷎品

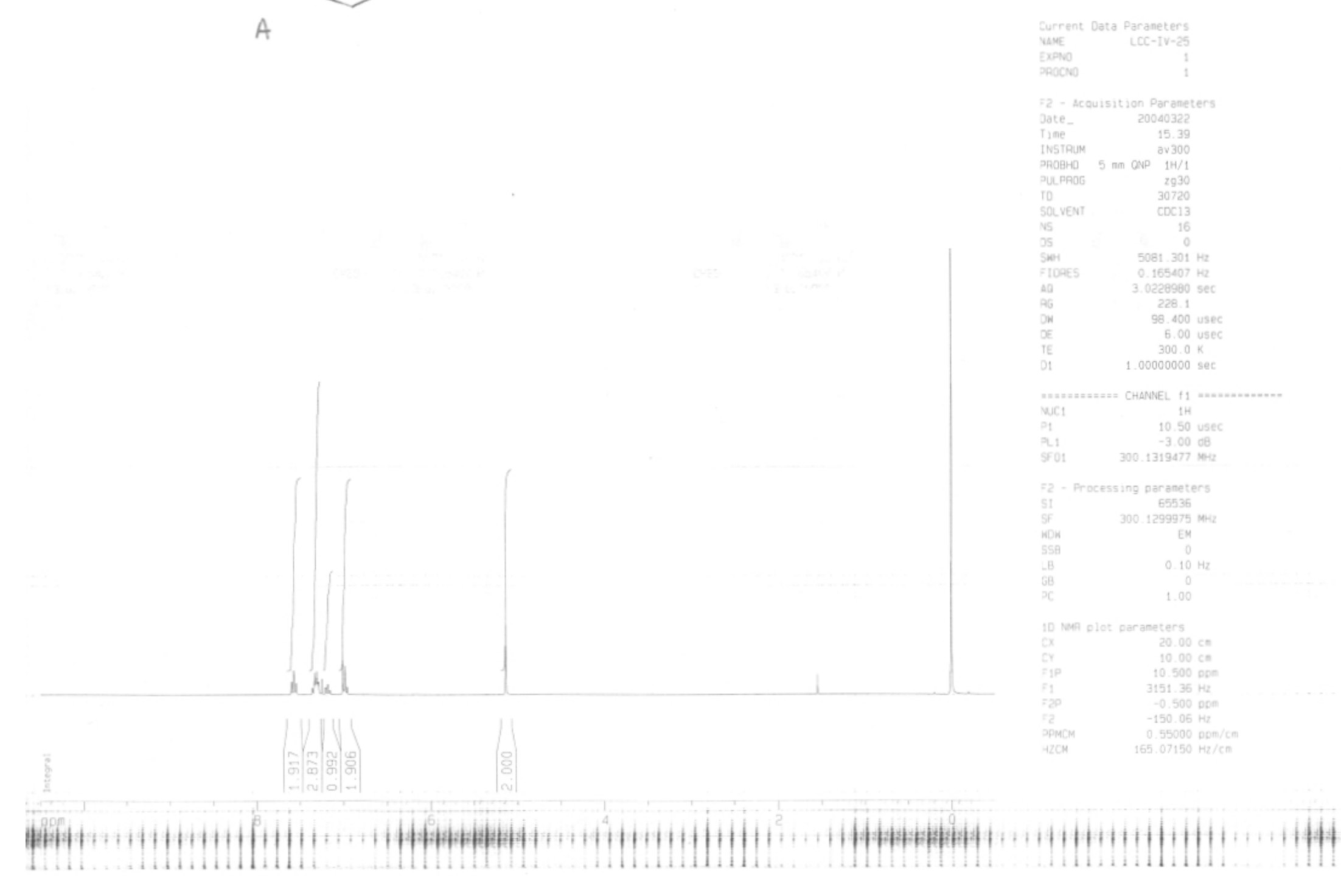




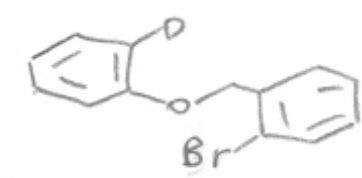

A

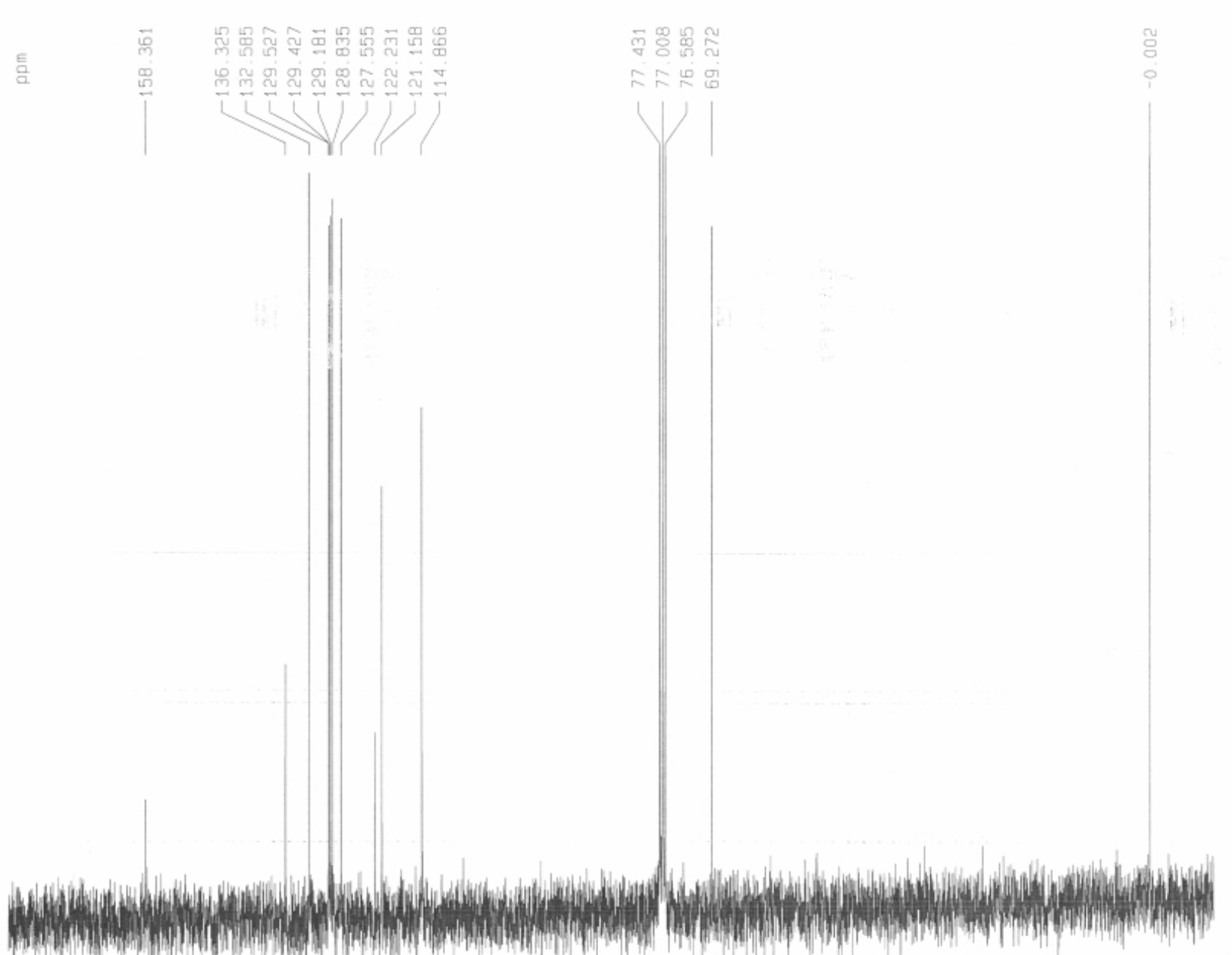

.

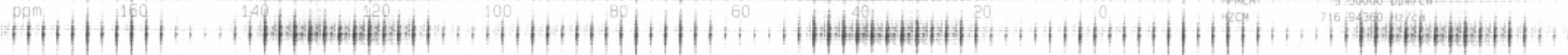

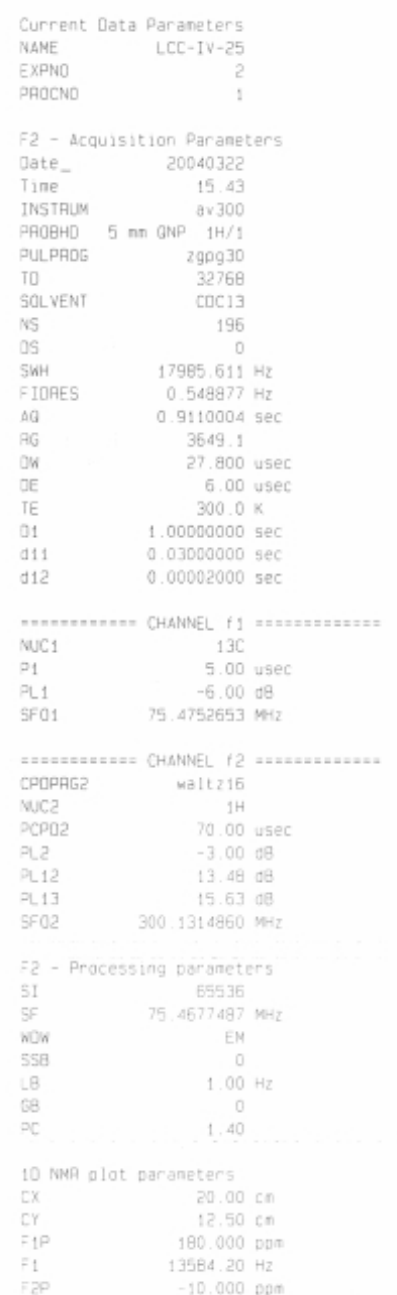




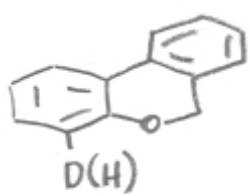

CRUDE

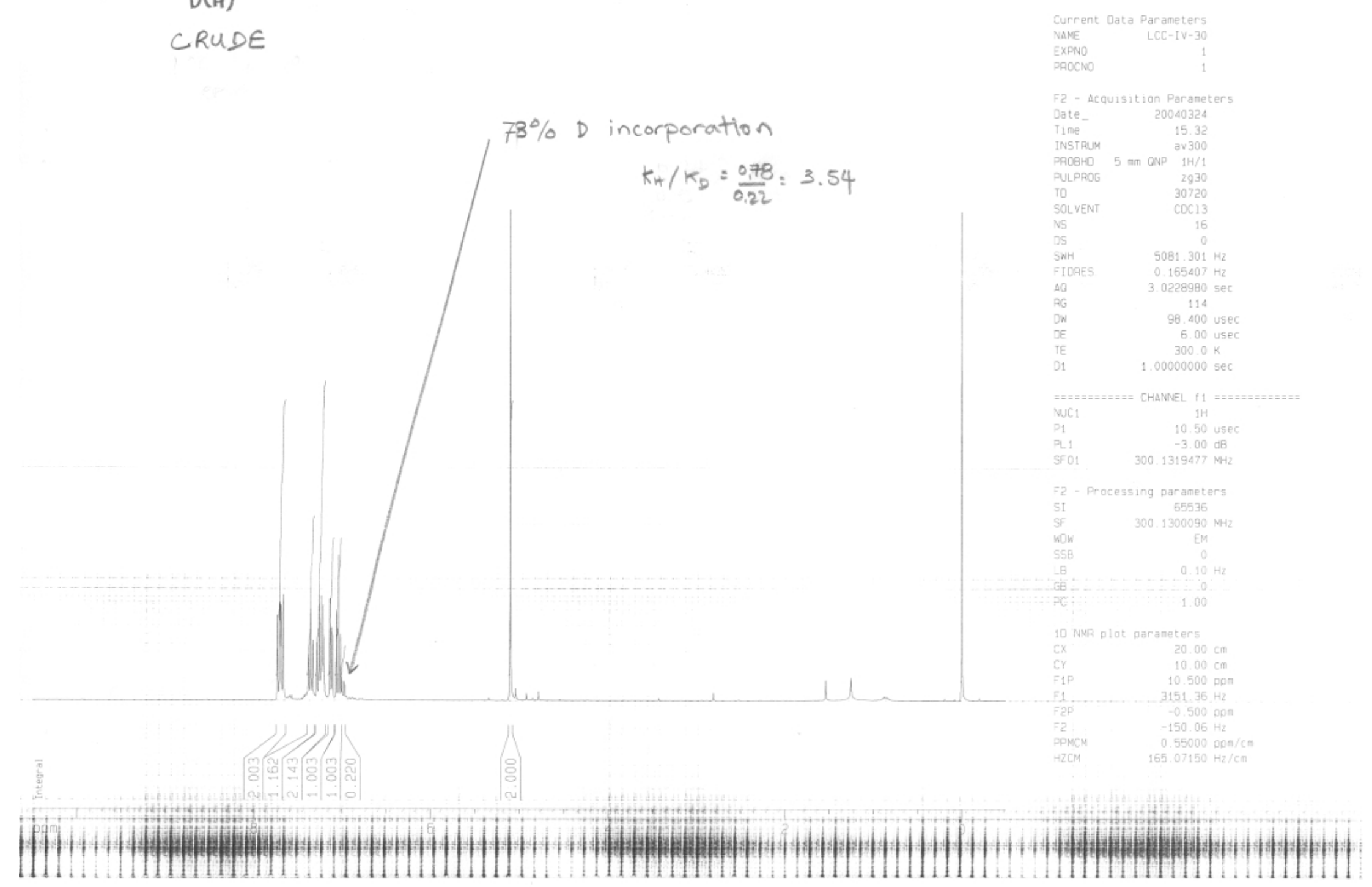




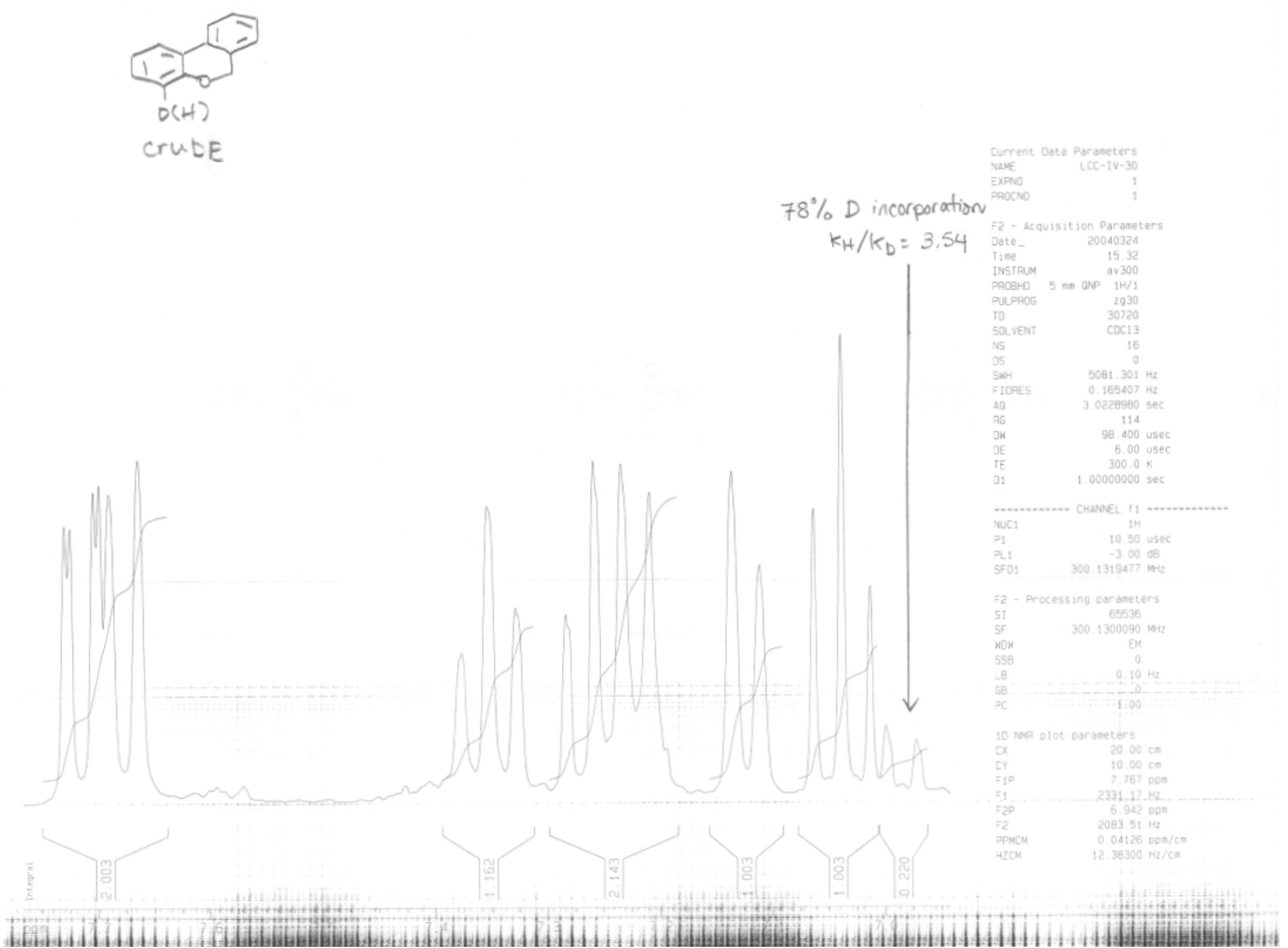

